Results: $27.3 \%$ of the all amputated patients or $41.5 \%$ of the non-traumatic patients were diabetic patients. Compared with non-traumatic amputation, patients with diabetic amputation were older $(65.4 \pm 11.6$ vs $50.1 \pm 20.4$ yrs, $p=0.000)$, with higher systolic blood pressure $(136.7 \pm 19.7 \mathrm{vs} 128.0 \pm 19.2 \mathrm{mmHg}$, $p=0.000$ ) and fasting blood glucose level ( $8.3 \pm 3.7$ vs $5.6 \pm 1.9 \mathrm{mmol} / \mathrm{L}, \mathrm{p}=0.000$ ), lower triglycerides $(1.3 \pm 0.7$ vs $1.5 \pm 1.0 \mathrm{mmol} / \mathrm{L}, \mathrm{p}=0.028)$ and high density lipoprotein cholesterol (HDL-C) (1.0 \pm 0.4 vs

$1.2 \pm 0.4 \mathrm{mmol} / \mathrm{L}, \mathrm{p}=0.000$ ) level. Minor amputation was more common in the patients with diabetic foot disease $(55.7 \%)$ than those with nontraumatic foot disease $(18.3 \%)\left(x^{2}=63.450, p=0.000\right)$. There were significant differences in days of hospital stay (33.5 vs $22.0 \mathrm{~d}$ ) and medical cost (36392 vs 25337 Yuan RMB) between the two groups. Conclusion: The $42.2 \%$ of non-tramatic amputation were caused by diabetic foot diseases The patients with diabetic amputation were older with higher blood glucose, blood pressure and lower TG and HDL-C level. Most of them required minor amputation. Their hospital stay was longer with significantly higher medical costs.

2440-P0

Management of Osteomyelitis in a Multidisciplinary Foot Clinic EDWARD B. JUDE, CHRISTINA ARAPOSTATHI, NIKOLAOS TENTOLOURIS, Ashtonunder-Lyne, United Kingdom, Athens, Greece

Diabetic foot infections and osteomyelitis are associated with substantial morbidity and mortality. The aim of this study was to look at the final outcome in patients with osteomyelitis.

In a retrospective study patients attending a diabetic foot clinic and diagnosed with osteomyelitis, were selected. All patients are followed up for five years after diagnosis of osteomyelitis. Diagnosis was based on a positive probe to bone test and an abnormal foot $x$-ray. Demographic, clinical, biochemical, treatment and outcome data were obtained from the computer records of the patients.

A total of 59 patients (51 with Type 2 diabetes, mean $\mathrm{HbA1c}: 7.9 \pm 1.9 \%$ and age: $67 \pm 14$ years) were entered into the study. The most frequent site of osteomyelitis was the forefoot (62.5\%). 12 patients underwent amputation $(75 \%)$, the majority of whom had osteomyelitis in the forefoot $(p=0.001) .31$ patients died during follow up and were older than those who survived (mean age: $71 \pm 13$ vs $62 \pm 14$ years, $p=0.009$ ). Risk factors such as neuropathy, ischemic heart disease, peripheral arterial disease and hyperlipidemia were more common among the amputated compared to the not amputated patients $(p<0.05)$ Patients who were younger healed more frequently compared to elderly patients (mean age: $61 \pm 15$ vs $70 \pm 13$ years, $p<0.005$ )

Age plays an important role in healing and mortality but site of osteomyelitis has a predictive role in amputations. Modifying the risk factors may help in reducing amputations and also more intensive management of the elderly patients might result in better outcomes in this age group.

\section{DIABETES EDUCATION}

2441-P0

Compliance With Lifestyle Recommendations and Achievement of Therapeutic Goals in Type 2 Diabetic Married Couples

IOANNIS IOANNIDIS, FOTINI ARTEMAKI, ATHANASIOS NIKOLOPOULOS, DANA TSALTA, DIMITRA TASIOPOULOU, NIKOLAOS KOMITOPOULOS, Athens, Greece

Introduction: The treatment of diabetes is based on lifestyle changes (diet and physical activity) and in compliance to medication in order to achieve the goals of good glucose control, lipids and blood pressure.

Aim: Was to examine the achievement of goals and lifestyle changes in diabetic couples and the degree of coincidence patterns among individuals in each pair.

Subjects: We studied 50 married couples of diabetic patients in reaching treatment goals and compliance in lifestyle changes and the degree of agreement between them

Results: $64 \%$ of couples had $\mathrm{HbA} 1 \mathrm{c}<7 \%, 8 \%$ had $>7 \%$ and the remaining $28 \%$ had one of the two $\mathrm{HbA} 1 \mathrm{c}<7 \%$.

$68 \%$ of couples had SBP $<140 \mathrm{mmHg}, 14 \%$ had $>140 \mathrm{mmHg}$ and $18 \%$ had SBP $<140 \mathrm{mmHg}$ one of the two.

As far as LDL cholesterol is concerned only $28 \%$ of couples had a value $<100.20 \%$ had $>100$ while in $52 \%$ of couples only one of the two achieved target value.

$68 \%$ of couples do not smoke, $8 \%$ smoke and in $26 \%$ of couples one of the two spouses smokes.

$80 \%$ of couples do not drink alcohol while in the remaining $20 \%$ one of the two spouses drink alcohol (low to moderate consumption).

$52 \%$ of couples do mild physical activity, $12 \%$ do not exercise at all and the remaining $36 \%$ reported physical activity of different intensity between the pair.

In $84 \%$ of couples referred daily consumption of more than 2 servings of vegetables, while only $8 \%$ do not eat any vegetables. Finally, $92 \%$ of couples consume at least 2 fruits daily and only $4 \%$ of couples do not eat fruit.

Conclusions: With a high degree of identity, people of couples with type 2 diabetes show good compliance in lifestyle change (mainly vegetables and fruit consumption and secondarily on physical activity and smoking habit). Highly identity between spouse recorded in good glycemic control and good blood pressure. The lowest degree of both achieving the goal and coincidence between the members recorded in LDL cholesterol.

Wait-List Control Study of a Diabetes (DM) Education Program

2442-P0 MATTHEW BERGER, SARAH G. IMERSHEIN, RICHARD A. JACKSON, Boston, MA

A wait-list control study ( $\mathrm{n}=89$ ) was used to assess biomarker change resulting from the use of On the Road (OTR), a low-literacy DM education and outreach program in Pennsylvania. OTR was a collaboration between Joslin Diabetes Center and Extension educators of the National Institute for Food and Agriculture, a division of the USDA. Programs were delivered to smal groups in non-medical settings, and included point-of-care $\mathrm{A} 1 \mathrm{C}$ and blood pressure (BP) testing, emphasizing the importance of $\mathrm{BP}$ control in reducing DM-related complications

Participants were randomized so that 46 completed OTR in February 2012 ("Intervention"), while 43 waited until June 2012 ("Control"). Spouse or companion pairs were allowed to randomize together as one unit, leading to some unevenly distributed characteristics, including systolic blood pressure (SBP). Follow-up rates were $87 \%$ for the Intervention group and $90 \%$ for the Control group.

Figure 1 shows mean SBP (average of 2 readings) and 95\% confidence intervals (Cl) for Intervention and Control participants at key study points.

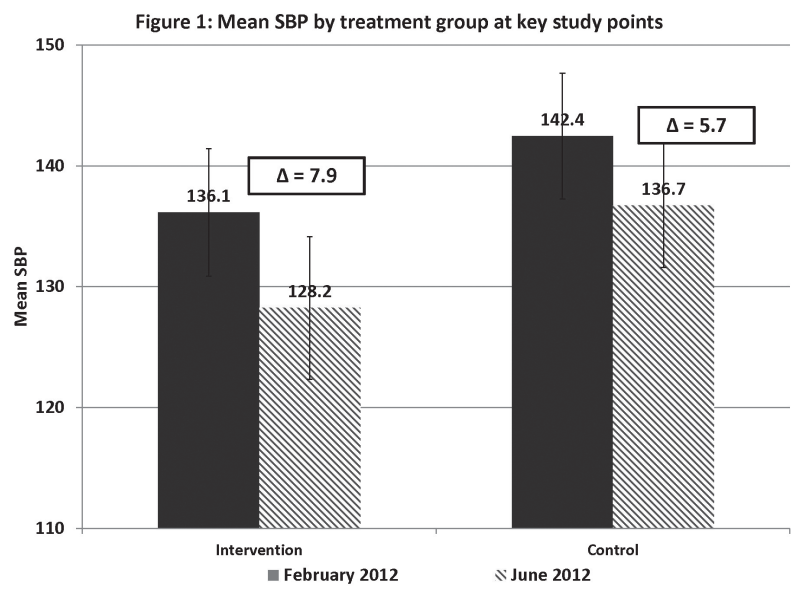

At baseline, mean SBP was 6.3 higher for Control participants than for Intervention participants ( $95 \% \mathrm{Cl}=-2.0$ - 14.6). However, mean SPB improvement was greater for the Intervention group than Controls (decrease of 7.9 versus 5.7, respectively, difference of difference $2.2,95 \% \mathrm{Cl}-4.1$ - 8.5).

Low-cost DM outreach education may lead to improved blood pressure control in low-literacy populations compared to those who do not receive education.

Supported by: U.S. Dept. of Agriculture

2443-PO

Improvement in Outcomes Following the Implementation of a Comprehensive Diabetes Management Program

SHELLEY WILLIAMS, MARGARET RIEDL, KENNETH IZUORA, Las Vegas, NV

The implementation of a comprehensive diabetes management program has been shown to improve outcomes in diabetes care. The establishment of a comprehensive diabetes education program in our center led to the introduc tion of diabetes self management education program and medical nutrition therapy taught through CDEs according to AADE 7 standards. Also, strategies for identification and correction of shortfalls in core measures in diabetes care were instituted. Point of care A1c testing and onsite retinal screening were initiated. There was also closer patient follow up between visits with more frequent contacts for review of home glucose logs and pump downloads We have analyzed hemoglobin A1c, LDL-C goals, BMI and compliance to dia- 


\section{DIABETES EDUCATION}

betic eye and foot exam guidelines before and after the implementation as part of our quality improvement initiative.

Out of all patients, 450 had follow up evaluations and were included in the analysis. Average age was 57 years, $50 \%$ were female and $88 \%$ had T2DM. Ethnic background; $49 \%$ Caucasian, 21\% Hispanic, 17\% African American, 5\% Asian and $8 \%$ other. There was a $10.6 \%$ reduction in A1c (from mean [SD] 8.5 [2\%] to 7.6 [1.6\%]). Mean LDL cholesterol improved from 93.6 to $85.2 \mathrm{mg} / \mathrm{dL}$ and the number of patients without documented foot and eye exams improved from $23 \%$ and $63 \%$ to $3.7 \%$ and $46 \%$ respectively.

Our review shows a positive impact on outcomes as a result of the successful implementation of a comprehensive diabetes management program. It also highlighted areas not meeting our goals that will be targeted for improvement. Our observation is consistent with the goals of establishing special diabetes programs that focus on the prevention and control of diabetes and its complications.

Supported by: DHHS; CDC

2444-P0

NEED (NExt EDucation): New Models and Tools for Participation and Dialogue in Diabetes Education

ULLA MøLLER HANSEN, GITTE ENGELUND, INGRID WILLAING, Gentofte, Denmark

The purpose of this study was to develop an innovative health education concept based on participation and dialogue in patient education.

As method of inquiry Design Thinking was used. Data on patient-perceived challenges in everyday life with a chronic illness was collected through four interactive workshops with 25 patients with chronic illnesses (type 2 diabetes, heart disease, and chronic obstructive lung disease). Cultural probes such as picture cards, quotes, and games were used with the dual purpose of collecting data and testing the potentials in using such tools in patient education. Furthermore we conducted interactive workshops with 28 health care professionals (HCP) performing patient education with two purposes: 1) translating the patient-perceived challenges into educational needs and 2) exploring the necessary competencies of HCPs for performing participation and dialogue in patient education based on the actual needs of the participants.

Data collection led to the development of a comprehensive health education concept for patient education called NEED consisting of two models and 24 tools: A model called The Balancing Personexpressing patient-perceived challenges in living with chronic illness and educational needs as defined by HCPs. Second a model for competency development in HCPs called The Juggler. Third a tool kit consisting of 24 health education tools supporting the two models in bringing patient needs and adequate educator skills into play.

In conclusion NEED constitutes an innovative and comprehensive approach to participatory patient education consisting of three separate yet combined components of patient education.

The concept has been feasibility tested in 49 clinical and community settings in Denmark. Results show that the concept enhances dialogue and participation and that HCPs find the tools easy and flexible to integrate in patient education

2445-PO

The Effect of a Self Management Programme Designed to Support Decision Making in People With Established Type 2 Diabetes HENRIETTA MULNIER, MARIA BARNARD, WENDA AITCHISON, ANNA REID, London, United Kingdom

Co-Creating Health (CCH) is a UK project that aims to improve collaborative decision making between patients with long term conditions and their health providers. It includes training for patients and health professionals. We focused on people with type 2 diabetes attending either primary, community or specialist services in four socially deprived inner city areas. Here we describe the effect of the patient Self Management Programme (SMP) on clinical outcomes.

Data were collected retrospectively from hospital and primary care database systems. An $\mathrm{HbA}_{1 \mathrm{c}}$ within 1 year prior to the programme start was accepted as baseline. A reading eight weeks after and within one year from starting the SMP accepted as post-programme. There were 166 people identified who fulfilled these criteria. Of those 76 were men $(46 \%)$, the mean age was 65-years (range 38-88, SD 11.1), mean diabetes duration 12.5 years (range $1-33$, SD 7.6), mean BMI was $31.8 \mathrm{Kg} / \mathrm{m}^{2}$ (range $20.4-50.8$, SD 6.6), and mean pre-SMP HbA $\mathrm{H}_{1 \mathrm{c}} 8.02 \%$ (range $4.0-16$, SD 1.83). There was a statistically significant drop in $\mathrm{HbA} 1 \mathrm{c}$ of $0.22 \%$ (95\% $\mathrm{Cl} 0.01-0.433) \mathrm{p}=0.049$ (mean $7.81 \%$ [SD 1.81]), and a small non-significant drop in weight of $0.6 \mathrm{Kg}$. In a subset of patients with a baseline $\mathrm{HbA}_{1 \mathrm{c}}$ of $>7.5 \%(\mathrm{n}=88)$ there was a larger impact with a statistically and clinically significant drop in $\mathrm{HbA}_{1 \mathrm{c}}$ of $0.58 \%(95 \% \mathrm{Cl} 0.24-$
$0.92) \mathrm{p}=0.001$ and a non significant weight loss of $0.8 \mathrm{Kg}(95 \% \mathrm{Cl}-0.36-2.027)$ $\mathrm{p}=0.170$. The results indicate that by promoting self management decision making, a clinically significant improvement in glycaemic control without weight gain can be achieved by individuals with comparatively long duration diabetes and in a socially deprived area.

2446-P0

Diabetes Education: Current Status in Polish Type 2 Diabetic Patients Receiving Insulin

KATARZYNA NABRDALIK, AGNIESZKA STRÓZIK, WłADYSłAW GRZESZCZAK, MAłGORZATA MASIEREK, JANUSZ GUMPRECHT, Zabrze, Poland, Ożarów Mazowiecki, Poland

In order to obtain good glycemic control patients treated with insulin have to be very well educated in self management of the disease and should possess a basic knowledge about the disease mechanisms and its possible complications. This study aimed to evaluate the status and source of diabetes education of type 2 diabetic patients treated with insulin and is a continuation of preliminary data coming from 2920 patients obtained in the year 2012. This was a 4-week observational study in the group that was currently enlarged up to 10309 patients ( $50 \%$ men; 50\% women; mean age of 63 years). The patients' general knowledge of diabetes, its complications and treatment have been assessed by the doctors with the use of a special questionnaire prepared for the purpose of the study and performed at the hospital and at out-patient clinics. Obtained outcomes confirmed the ones presented in preliminary data. General knowledge about diabetes was assessed to be very good only in 1,9\% (2\% in preliminary data), sufficient enough in $40,1 \%(39.5 \%$ in preliminary data), and inadequate in $8.5 \%$ (8.6 \% in preliminary data) of studied patients. The main source of information about diabetes came from doctors $193.8 \%$ vs. $92.7 \%$ in preliminary data) and nurses $(76.2 \%$ vs. $77.3 \%$ in preliminary data) and educational materials $(39.9 \%$ vs. $44.6 \%$ in preliminary data). $65.6 \%$ of patients (vs. $66 \%$ in preliminary data) expressed intention to increase their knowledge about the disease and preferred to obtain information from doctor ( $95.2 \%$ vs $95.1 \%$ in preliminary data) and educational materials ( $60.3 \%$ vs $64.5 \%$ in preliminary data). For $68.4 \%$ (vs. $60.1 \%$ in preliminary data) patients recognized authority with reference to knowledge about diabetes was a doctor and for $19.4 \%$ (vs. $26.7 \%$ in preliminary data) nurse. An actual low level of diabetes knowledge among polish type 2 diabetic patients receiving insulin therapy suggests that there is a significant need for improvement of patients diabetes education.

2447-P0

\section{WITHDRAWN}


2448-PO

Longitudinal Preferences in the Format of Continuing Medical Education (CME) Activities in Diabetes, 2006-2011

MARGERYJ. TAMAS, ANGELAC. MCINTOSH, AMARYNTHIACARBONARA, STEVEN A. WEINMAN, Atlanta, GA, Somerset, NJ

CME activities are offered in a variety of formats, including live, print, and electronic media. Electronic media may include audiotape, videotape, CDROM, personal digital assistant (PDA), smartphone, tablet computer, or Internet. Some marketing surveys suggest high demand for CME activities on newer electronic devices, especially smartphones.

A retrospective review of activity evaluation data was conducted. Activity format preferences were indicated in evaluation summaries for 15 live meetings on the clinical management of diabetes during 2006-2011, all of which were certified for physicians, including primary care providers and diabetes specialists. Participants in the educational activities made 1 or more unranked selections of format preferences from a list provided. The total number of participants making each selection was calculated as a percentage.

Low longitudinal correlations were observed for live activities $\left(r^{2}=0.1201\right.$, $r=.35)$ and CD-ROMs $\left(r^{2}=0.1603, r=0.40\right)$ during 2006-2011. Preference for live activities was consistently high and increased. Preference for CD-ROMs was consistently low but increased. Preference for print and newer electronic formats such as podcasts, PDAs, and smartphones was uniformly low (all $r$ $<.30)$.

Attendees of live diabetes CME activities increasingly prefer live activities and do not exhibit a high or increasing preference for activities in newer electronic formats, including smartphones.

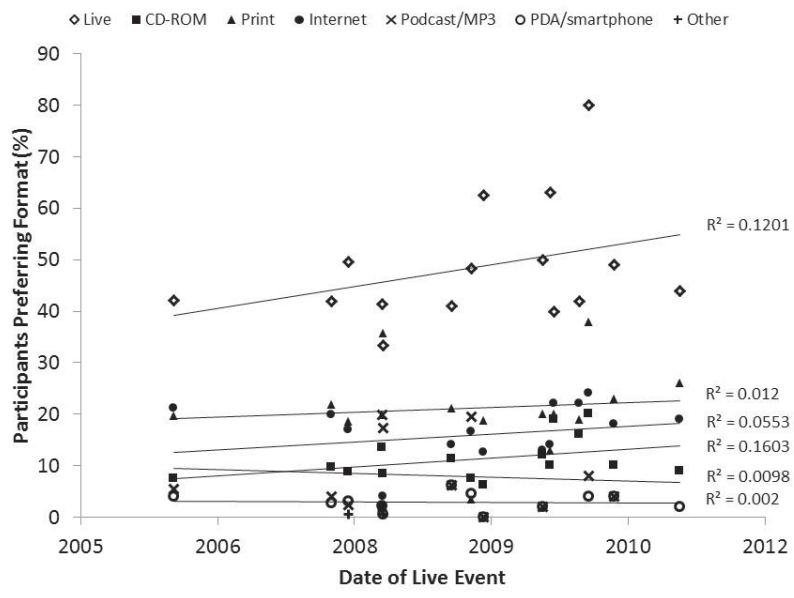

2449-PO

Structured Feedback in the Development of the First Educational Smartphone Application (Pumps4Kids) for Insulin Pump Starts COLIN P. HAWKES, PETER C. HINDMARSH, MARIAN MCCARTHY, STEPHEN M.P. O'RIORDAN, Cork, Ireland, London, United Kingdom

Background: The transition from subcutaneous injections to CSII requires extensive education for parents and carers. Increasing smartphone use by children may provide an opportunity to support this education. We describe the process of developing the first smartphone app to enhance pump start education, utilizing extensive patient feedback.

Methods: Patients received daily phonecalls for one week immediately following pump education and CSII commencement. Difficulties experienced were used to guide app development. Patient experience was further integrated into application design using a) focus group of teenagers established on CSII and b) questionnaires integrated into the app.

Results: 20 children 12male (11-16 years) underwent pump start via a structured Pump School. Over 300 phonecalls were documented for the first 7 days in all children. These identified hypoglycemia (mean 9.7 (SD 5.2) over 7 days) and hyperglycemia requiring pen injection (mean 1.2 (SD 1.9) over 7 days). Other difficulties included set changes, sick-day rules and exercise induced hypoglycemia. A focus group $(n=10)$ identified further areas for improvement. Ongoing integrated feedback will be updated at 3 monthly intervals. A smartphone app was created. This included 20 pages, 3 calculators, and modifiable standard unit settings for the European and American patients. This app was launched in January 2013 and is free of charge.

Conclusion: This is the first educational app for insulin pumps targeted at children. Problem orientated design and extensive structured feedback played key roles in developing this age-appropriate educational tool for children and teenagers with diabetes.

2450-PO

Multidisciplinary Individual Team Approach Effective to Delay eGFR Decrease in Patients With Diabetic Nephropathy (DN) even in a Short Period

MAMIKO HITOMI, TERUKO SATO, YUKO KAWAKAMI, TATSUMI MORIYA, Sagamihara, Japan

DN is the most prevalent cause of end stage renal disease requiring hemodialysis (HD). This service, covered by the national health insurance, authorized intensive care provided by the team approach, including doctors, registered nurses and dietitians, that aimed to prevent or delay the induction of $\mathrm{HD}$ in Japan from April 2012 at which time, we began intensive care and individual education by three professionals. The team approach was preformed for 33 DN outpatients. Fifteen patients ( 8 men, age $67 \pm 8$ y) who had multiple educational courses were analyzed for the present study. The observation period was from April to September, 2012. Medical nutrition therapy was conducted according to Japanese guidelines. Daily energy was set at $30-35 \mathrm{kcal} / \mathrm{kg}$ ideal body weight (IBW), protein was $0.6-0.8 \mathrm{~g} / \mathrm{kg} \mathrm{IBW}$, and sodium was $6 \mathrm{~g} /$ day. The number of the intervention was 3.1 1.1 times/a patient during the observation. Estimated GFR (eGFR) did not change between baseline $(31.4 \pm 11.8 \mathrm{~mL} /$ $\left.\mathrm{min} / 1.73 \mathrm{~m}^{2}\right)$ and follow-up $(30.6 \pm 12.0)$ and the eGFR inclinations were -0.32 $\mathrm{mL} / \mathrm{min} / 1.73 \mathrm{~m}^{2} / \mathrm{month}$, which were significantly slower than those without the team approach $(-1.08)(\mathrm{p}<0.05)$. Urinary albumin was significantly improved from 886 (32.7-5121.6) mg/gCr to 532 (46.8-3953.6) ( $\mathrm{p}<0.0001)$. HbA1c was $7.5 \pm 1.4 \%, 7.1 \pm 1.5$ and $B P$ was $137 \pm 12 / 78 \pm 9 \mathrm{mmHg}, 139 \pm 12 / 75 \pm 10$ at baseline and follow-up, respectively. The estimated daily protein and sodium intake using 24 -hour urine collection specimens were $1.13 \pm 0.66 \mathrm{~g} / \mathrm{kg} \mathrm{IBW}, 0.99 \pm 0.31$ and $8.8 \pm 4.7 \mathrm{~g} / \mathrm{day}, 8.2 \pm 4.8$, at baseline and follow-up, respectively. Even in a short observation period, eGFR decline in patients with intensive and individual education with 3 professionals was slower than that for patients with no education. The individual intensive education provided by the team approach was effective for DN self-management, resulting in improving eGFR decline and anticipated the prevention or delay of $\mathrm{HD}$ induction.

2451-PO

Understanding the Standard of Care in the Treatment of Type 2 Diabetes in China: Results from a National Survey

LINONG JI, JULIE NEWMAN, Beijing, China, Mulgrave, Australia

Treatment practices for the management of type 2 diabetes (T2D) have evolved considerably in the past decade. In coordination with the Chinese Diabetes Society (CDS), we developed a quantitative survey to explore the standards of care for T2D in China. Such assessment is particularly relevant to China, given that the T2D epidemic continues to overwhelm the healthcare system.

A national online survey of physicians involved in the management of T2D in China was conducted over a 4-week period in 2012. Completed responses were obtained from 1028 physicians across different regions, hospital grades, specialties and professional rankings.

Overall, $83 \%$ of surveyed physicians were at least "aware" of the CDS guidelines on standards of care for T2D. Level of awareness was directly related to hospital grade, specialty, geographic location, professional rank and participation in CDS training. The consistency and frequency of practice in accordance with CDS guidelines on standards of care for T2D was also found to differ according to these variables. Gaps in knowledge were identified and plans to increase awareness, compliance and improve the overall standard of care are underway.Understanding of the variables affecting the management of T2D in China will help CDS further identify unmet needs that can be addressed to improve and standardise the provision of healthcare services, impact the early diagnosis and treatment of T2D, and stem the diabetes epidemic.

Supported by: Bristol-Myers Squibb

2452-PO

Developing a Rating Scale for Blood Glucose Meter Display Readability

WILLIAM B. REUSCHEL, MARK USLAN, Huntington, WV

The American Foundation for the Blind has developed a rating scale for blood glucose meters (BGM) which represents the readability of information on the display. The purpose of this rating scale is to provide consumers with practical information about the readability of a device based on physical characteristics of the display such as contrast, reflection properties, and font information. A user study was conducted to validate the concept of the rating 


\section{DIABETES EDUCATION}

scale and tune its parameters. Ten fully sighted individuals and ten diabetic individuals who were visually impaired but still used their vision for reading were asked to perform the following tasks:

1. Answer interview questions regarding age, corrective lens usage, etc.

2. Arrange eight $\mathrm{BGMs}$ in order of readability in room lighting

3. Choose the more readable BGM in a series of five pairings

4. Take a near visual acuity test and a near contrast sensitivity test

5. Perform tasks 2 and 3 again under a lamp simulating sunlight

6. Answer exit questions designed to determine what factors the participant used to make decisions about readability.

All of the participants from both groups were able to read the large blood glucose reading displayed on the devices. None of the fully sighted participants struggled with the small text (such as time and unit labels) but many of the visually impaired individuals did struggle with this text. The main factors that the participants identified when making comparisons were font size, contrast, boldness/thickness of the numbers, amount of information displayed, and size of the display. Two factors that were identified that could contribute to misreading the display were "shadow" segments on some displays which were visible at certain viewing angles even when the segments were turned off and numbers which were too close to an edge. The rating scale which is based on measured contrast, reflection, and font size properties was tuned to coincide with the rank-order data collected during the investigation.

Supported by: Jesse Ball duPont Fund

2453-P0

Eat What You Love With Diabetes: How to Use the Mindful Eating Cycle for Self-Management

\section{MiCHELLE MAY, MEGRETTE FLETCHER, Phoenix, AZ, Dover, NH}

This session will review the benefits of mindful eating and how this ancient technique can be used to improve blood sugar control through hunger and fullness awareness training. Mindful eating has been shown to improve meal satisfaction, decrease binge eating, decrease non-hunger eating, promote sustained weight management, and improve blood sugar control. Recent research compared mindful eating training to DSMT and found significant changes in eating behaviors and reductions in A1C. However, clinicians sometimes have difficulty conveying mindful eating concepts to their patients. This barrier can be overcome by using the Mindful Eating Cycle, a simple cognitivebehavioral model. This practical tool explores six fundamental questions for conscious decision making: Why do I eat? When do I eat? What do I eat? How do I eat? How much do I eat? and Where do I invest my energy? The Mindful Eating Cycle helps clients become more mindful about their intention and foster their ability to direct their attention when eating and planning meals. The session will focus on how this model can be adapted to teach DSMT, $\mathrm{BGM}$, and lay the necessary groundwork for lifelong learning about diabetes.

2454-P0

Language, Media Use and Health Behavior in Inner-City Latino Diabetics and Caregivers

AYESHA SHAIKH, MARIA MILAN, ERIDA VASOUEZ, FRANCES SILVA, MARIANO REY, BALAVENKATESH KANNA, Bronx, NY, New York, NY

Inner-city Latino New Yorkers (LNY) have high burden of diabetes. Health education is important in diabetes control. This is a pilot survey of Latino diabetics and their caregivers in Bronx, New York (NY) to study language and media use.

Methods: Demographic, ethnic, language, education, health behavior data were collected. Descriptive measures are presented. The impact of language on use of media (news, radio, television (TV) and internet) and health behaviors was analyzed.

Results: Of 82 subjects, $65(79 \%)$ were women. Median age was 58.6 years (range 24-81 years); 76 (92.7\%) Latinos; 71 (86.6\%) foreign-born; 60(73.2\%) unemployed \& $13(15.8 \%)$ went to high school. $65(79.3 \%)$ have health insurance; $70(85.4 \%)$ a primary care provider \& 79 (96.4\%) visited doctor's office in the last 1 year. $67(81.7 \%)$ had Spanish as their primary language. $41 \%$ speak, read and write in English fluently. $30(37 \%)$ read English news and 52(63.4\%) in Spanish. 47 (57\%) watched English TV \& 67(81.7\%) watch Spanish TV. 31 (37.8\%) listened to English radio \& $50(61 \%)$ to Spanish radio. 16 (19.5\%) used English on internet\& 14(17\%) used Spanish. Only 20 (25.4\%) used media to affect a decision about treating their medical condition, \& 38 (46.3\%) reported a change in the way they think specifically about diet and exercise. Adjusted for above factors, treatment decision and understanding risks was associated with use of Spanish media. (Odds ratio 6.59, 95\% confidence interval 1.1-39.5, $\mathrm{p}$ value $=0.039$ )
Discussion: Our pilot study shows inner-city LNY are mostly foreign born, read write \& speak Spanish language, and use Spanish media. Health behavior change correlated with use of Spanish media. These findings provide insight into use of language and media in public health and patient education programs in the NY Latino community.

Supported by: New York University

2455-P0

Cities for Life: Mydiabetesconnect.com: A Mixed-Methods Approach to a Community Diabetes Resource Website

ANDREA L. CHERRINGTON, APRIL A. AGNE, DEBBY M. LACRUZ, ALFREDO L. GUZMAN, ALEXANDER COTTER, JANETP. THURMAN, NATALIA LOSKUTOVA, EDWIN B. FISHER, Birmingham, AL, Leawood, KS, Chapel Hill, NC

Finding programs to help individuals manage their diabetes in real-time can be a challenge for both patients and providers of care. The objective of this project was to develop a website to help individuals identify and locate existing resources in their community to facilitate diabetes self-management and lifestyle modification. The site was developed as part of a larger city-wide diabetes management program, "Cities for Life" in Birmingham, Alabama. We used a mixed-methods approach, including semi-structured interviews with key members of local community-based organizations and the local safety-net hospital combined with community-wide outreach to identify programs. Semistructured interviews focused on understanding local resources and barriers to their utilization. Resources and organizations were identified using a snowball approach and word-of-mouth. Potential resources were contacted to confirm information and willingness to be listed on the website. Key informant interviews $(n=8)$ confirmed need for a local community resource database, desire for up-to-date information and interactive components. Key barriers included: transportation, affordability, internet access and a need for basic diabetes education. Based on these data, we used an iterative approach to design an interactive website that identifies over 200 resources in all the major categories related to diabetes management, including diet; physical activity; diabetes education and support; and stress management. New resources can be uploaded at any time and a star-rating system allows users to assess each resource. The site allows users to locate resources and events as well as facilitate user communication with others in their community through a professionally monitored message board. Although content is specific to Birmingham, the development process and site structure are readily generalizable to other communities.

Supported by: Sanofi U.S.

2456-PO

The Effects of Exercise Education With Accelerometer on Glucose Control, Lipid Profile and Obesity in Type 2 Diabetic Patients YEO-JIN MOON, SUNG-WOO PARK, KI-WON OH, WON-YOUNG LEE, CHUL-YOUNG PARK, EUN-JUNG RHEE, SE-EUN PARK, Seoul, Republic of Korea

Aims: The purpose of this study examined what effect did it have on glucose control, lipid profile and obesity of type 2 diabetic patients to make a difference in exercise education method by using accelerometer lifecorder that could monitoring physical activity.

Methods: The subjects participating in the study were type 2 diabetic patients $(n=16)$ who were divided into two groups: experimental group $(n=9)$ educated about total energy expenditure, total steps and physical activity with lifecorder (suzuken co., Nagoya, Japan) and control group (n=7) not educated about energy expenditure, total steps and physical activity. Each exercise education program was implemented for 1 session per month, over 3 months.

Results: Considering change pre- and post-exercise education, FBS $(156.0 \pm 35.6$ vs $114.6 \pm 13.8 \mathrm{mg} / \mathrm{dL}), 2 \mathrm{hPPG}(224.2 \pm 28.4$ vs $156.3 \pm 25.0 \mathrm{mg} / \mathrm{dL})$, $\mathrm{HbA}_{1 \mathrm{c}}(7.2 \pm 0.7$ vs $6.5 \pm 0.6 \%)$, total-ch $(198.2 \pm 48.3$ vs $153.2 \pm 30.5 \mathrm{mg} / \mathrm{dL})$, TG $(173.8 \pm 140.6$ vs $116.7 \pm 45.3 \mathrm{mg} / \mathrm{dL}), \mathrm{BMl}\left(29.9 \pm 4.2 \mathrm{vs} 27.4 \pm 3.8 \mathrm{~kg} / \mathrm{m}^{2}\right)$ and waist circumference $(96.6 \pm 10.1$ vs $90.2 \pm 9.0 \mathrm{~cm})$ showed significantly decreasing tendency and $\mathrm{HDL}-\mathrm{C}(46.9 \pm 14.0 \mathrm{vs} 53.3 \pm 12.0 \mathrm{mg} / \mathrm{dL})$, total energy expenditures $(2122.2 \pm 335.0$ vs $2402.9 \pm 347.6$ kcal), physical activity $(225.8 \pm 57.4$ vs 404.3 $\pm 67.2 \mathrm{kcal})$, total steps $(6906.1 \pm 2024.0$ vs $10975.4 \pm 1772.6$ steps $)$ showed significantly increasing tendency in experimental group.pp2 $(254.4 \pm 23.5 \mathrm{vs}$ $223.6 \pm 38.0 \mathrm{mg} / \mathrm{dL}), \mathrm{TG}(154.3 \pm 16.6$ vs $153.6 \pm 17.2 \mathrm{mg} / \mathrm{dL})$ and BMI $(32.1 \pm 2.4$ vs $31.1 \pm 2.3 \mathrm{~kg} / \mathrm{m} 2)$ showed significantly decreasing tendency, and only total steps (5393.1 1616.7 vs $7115.0 \pm 1361.1$ steps) showed significantly increasing tendency in control group.

Conclusions: In this study, we found that exercise education with accelerometer may be useful in improving glycemic control, lipid, obesity and physical activity with type 2 diabetic patients. And using equipment as education tool alone may be useful in improving physical activity and obesity. 
2457-PO

The PRIDE (PaRtnering to Improve Diabetes Education) Toolkit KATHLEEN L. WOLFF, LAURA C. CHAMBERS, STEFAN BUMOL, BECKY P. GREGORY, DIANNE DAVIS, RUSSELL ROTHMAN, Nashville, TN

Low literacy materials and clear health communication can improve diabetes (DM) outcomes. We developed a novel literacy and culturally sensitive diabetes education toolkit (PRIDE) and are testing it in a cluster randomized trial (RCT) in ten state health department clinics.

We developed 24 educational modules to promote diabetes education and shared goal setting between providers and patients. Materials cover common self-care skills including medications, monitoring, nutrition, exercise, tobacco, and depression. Materials were developed by experts in diabetes, diabetes education, health behavior, and other areas. The toolkit builds on our previously validated Diabetes Literacy \& Numeracy Education Toolkit. Materials were translated and culturally adapted for Spanish patients. Cognitive feedback was solicited from patients and providers. Two independent raters evaluated the English materials using the Suitability Assessment of Materials (SAM) approach. Reading level was assessed using the Flesch Kincaid Coleman-Liau, SMOG, FRY, and ARI. Mean scores for SAM and reading level for each module was determined.

To date, $378 \mathrm{DM}$ patients have been enrolled. Intervention patients have mean education 11.2 yrs, $81 \%$ with adequate literacy (STOFHLA) , mean Diabetes Numeracy Test score 60\%, 19\% are Latino, and mean A1C of $9.7 \%$. Mean SAM score for the modules was $91 \%$ (SD 5.6, range $74-98 \%$ ), mean readability grade level is 5.2 (SD 1.1, range 3.2-7.2). Providers are most frequently using materials that address general DM information, monitoring, foot care, basic nutrition, Plate Method, snack foods, and log sheets.

We successfully developed 25 novel educational modules for diabetes selfmanagement education and support. Modules cover a wide range of diabetes related activities and are suitable for English and Spanish speaking patients with lower literacy. In the RCT, we will evaluate the impact of the PRIDE materials and provider communication training on patient knowledge, behavior, and clinical outcomes.

2458-PO

Effect of Educational Intervention on Metabolic Control in Type 2 Diabetic Patients attending Government Medical College, Kozhikode, Kerala, India

G.K. SREEHARI, R. CHANDNI, N.K. THULASEEDHARAN, Kozhikode, India

Diabetes is a chronic disease. The role of the health care provider is to serve as a coach to the patient, who has primary responsibility for delivery of daily healthcare. Our aim was to study the effect of educational intervention on metabolic control in type 2 diabetic patients in diabetes clinic, Kozhikode Medical College.190 subjects of type 2 diabetic patients of duration $<5$ years were randomly selected and assigned randomly to intervention and control group. Baseline metabolic parameters were assessed initially in all. Educational intervention was given to 96 subjects who belonged to the intervention group, including 45 minutes of interactive session, 10 minutes of diet and lifestyle counseling, and two educational booklets. Monthly reinforcements were given for the following 6 months, in the intervention group. The routinely practiced diet \&amp; lifestyle counseling and drug treatment were continued in both the groups. Metabolic parameters reassessed at the end of 6 months in both intervention and control groups. Statistically significant favourable change in diet modification, exercise pattern, body weight, BMI, $B P, F B S, P P B S, H b A 1 C$, and lipid values observed in subjects of intervention group as compared to control group, at the end of the study. The p values for the difference in change after intervention in BMI,BP,FBS,PPBS and HbA1c were $<0.001$. Diabetic subjects in this study were having high Total cholesterol and high LDL as against the commonly described high TGL and low HDL. We observed that the educational intervention and monthly reinforcement of the intervention are effective if given along with the routine education and pharmacotherapy for the metabolic control in Type 2 diabetic patients attending Government medical college, Kozhikode.
2459-P0

The Baseline Data of Enrolled Patients With Type 2 Diabetes in OPENING (Organization Program of DiabEtes INsulIN ManaGement) Study

JUMING LU, LINONG JI, XIAOHUI GUO, JIE LIU, Beijing, China, Taiyuan, China

Purpose: A 16-week, open-label, multicenter, prospective, randomized, controlled study of Structured Education in T2DM patients with insulin treatment, referred as Organization Program of DiabEtes INsulIN ManaGement (OPENING) study, was designed to evaluate the effectiveness of structured education in patients with T2DM. This is to summarize the baseline data of enrolled patients with type 2 diabetes in OPENING study.

Methods: Eligible patients were over 18 years of age and inadequately responded to two or more oral anti-diabetic treatments (OADs) for over 3 months $(\mathrm{HbA} 1 \mathrm{c} \%>7.5 \%)$. Patients were randomized (1:1) into the education group (structured education plus insulin therapy) and the control group (usual care plus insulin therapy). The baseline data of enrolled patients were collected and statistical analysis was performed. Results: Totally 1511 patients with T2DM were enrolled from 48 hospitals across China, of which $50.10 \%$ was male and $49.90 \%$ was female, with a mean age of $57.15 \pm 10.55$ years, a mean BMl of $24.88 \pm 5.75 \mathrm{~kg} / \mathrm{m}^{2}$, and a mean diabetic duration of $7.76 \pm 5.61$ years. There were $338(22.40 \%)$ patients suffered from complications of diabetes, that included diabetic peripheral neuropathy (38.37\%), diabetic retin opathy $(29.53 \%)$ and diabetic nephropathy (12.56\%). There were 720 (47.56\%) patients with comorbidities, of which $49.18 \%$ was hypertension, $19.78 \%$ was dyslipidemia, $11.06 \%$ was coronary heart disease, and $4.17 \%$ was cerebrovascular disease. The mean value of baseline $\mathrm{HbA1c}$ and fasting blood glucose were $9.40 \pm 1.92 \%$ and $10.09 \pm 3.26 \mathrm{mmol} / \mathrm{L}$, respectively.

Conclusion: The enrolled patients in OPENING study had a high glucose level, and high prevalence of diabetic complications and comorbidities. It means that in China the initiation of insulin treatment is relatively late, and the overall glycemic control still needs to be improved.

2460-PO

A Performance Improvement CME Intervention With Diabetes Preceptorship as a Model for Collaborative Institutional Learning DAVIDA F. KRUGER, VIRGINIA VALENTINE, INTELLIGENT MEDICAL DECISIONS, INC. PRECEPTORSHIP WORKING GROUP, Grosse Pointe, MI, Albuquerque, NM Media, PA

Diabetes care is complex,many multiple therapeutic agents,difficulty achieving and maintaining glycemic control,progression of the disease, barriers posed by patient and healthcare team to successful long-term management.These complexities suggest innovative,collaborative preceptorship approach to professional education would contribute to better patient outcomes.Performance improvement continuing medical education intervention with a diabetes preceptorship model was developed to enhance education of diabetes treatment team members regarding management options, with the ultimate goals of improving patient adherence,outcomes and improving the quality of care delivered.The program includes $1 / 2$ day of education,email/ telephone contact and conference calls between preceptor and clinical sites. $A$ clinic champion was identified to work with preceptors.Patient goals included the reduction of glycated hemoglobin (A1C),weight loss,decreased hypoglycemia, and reduced nausea.With regular preceptor follow-up,team members became proficient in the target areas of patient management, and used this knowledge to improve patient care.This successful educational format was for management of patients receiving adjunctive Amylin therapy.However, this model of education, with an emphasis on regular follow-up and mentor support, could serve as an effective example for clinician education across all aspects of diabetes care. While data are still forthcoming,initial findings suggest that the program has resulted in improved patient outcomes, including a reduction in A1c and weight loss. Between March 2012 and November 2012 7 clinics completed data on 20 patients, baseline A1c of $8.5 \pm 0.8 \%$, mean weight of $246.6 \pm 28.6 \mathrm{lbs}$. After 5 follow up visits A1c reduction was seen in 11 patients, and weight loss in 16.7 patients reached $A 1 c$ of $<7 \%$ or less. Patients achieved a final A1c reduction of $3.73 \%(p=.075)$ and weight reduction of $-2.45 \%(P<0.001)$

.




\section{DIABETES EDUCATION}

2461-PO

Self-Monitoring of Blood Glucose in Subjects With Newly Diagnosed Type 2 Diabetes after Short-Term Continuous Subcutaneous Insulin Infusion

AILING CHEN, ZHIMIN HUANG, WANPING DENG, LICHENG LI, XIAOJUAN ZHAO, XUESI WAN, YANBING LI, Guangzhou, China

To follow and investigate the self-monitoring of blood glucose (SMBG) in subjects with newly diagnosed type 2 diabetes after short-term continuous subcutaneous insulin infusion (CSII) and to examine the relationship between SMBG and $\mathrm{HbA}_{1 \mathrm{c}}$. A total of 118 newly diagnosed type 2 diabetic subjects who have gained clinical remission (maintaining euglycemia) after short-term CSII were directed how to manage diabetes and followed up after being discharged. All subjects completed the survey about SMBG practice and the changes in SMBG frequency at the baseline, 3rd, 6th, and 12th month along with measurement of $\mathrm{HbA}_{1 \mathrm{c}}$. 65 subjects (remission group) maintained good glycemic control (FBG $<7 \mathrm{mmol} / \mathrm{L}, 2 \mathrm{hPG}<10 \mathrm{mmol} / \mathrm{L}$ ) for more than one year, and the others (nonremission group) were shorter. The baseline characteristics of both groups were comparable except for the educational attainments, FBG and $2 \mathrm{hPG}$. The proportions of subjects who can insisted SMBG in remission group $(73.8 \%, 63.1 \%)$ were significantly higher than those in nonremission group at the $6^{\text {th }}$, and $12^{\text {th }}$ month $(45.3 \%, 30.2 \%, P<0.01)$ but not at the baseline(95.4\% VS. $86.8 \%, P>0.05)$ and $3^{\text {rd }}$ month(87.7\% VS. $\left.79.2 \%, P>0.05\right)$. Significantly greater proportions of subjects about the SMBG frequency(How often a week) in remission group achieved throughout the follow up than those in nonremission group $(p<0.001)$. Different SMBG frequency could significantly collected with different $\mathrm{HbA1C}$ at the $6^{\text {th }}(0$ day/week $(7.09 \pm 0.93)$ vs. 1day/ week(6.88 \pm 0.79$)$ VS. 2 days/week $(6.30 \pm 0.66)$ VS. 3days/week( $5.97 \pm 0.51)$. $P<0.05)$ and $12^{\text {th }}$ month $(7.12 \pm 0.72$ VS. $6.82 \pm 0.78$ VS. $6.25 \pm 0.44$ VS. $6.23 \pm 0.80$, $P<0.05)$. The follow up data showed that in newly diagnosed type 2 diabetic subjects after short-term CSII, better self-monitoring of blood glucose might be beneficial for better glycemic control.

\section{Supporting Young Adult Diabetes Transitions}

2462-P0

\section{NICOLE JOHNSON, STEPHANIE MELTON, Tampa, FL}

Young adults with diabetes face enormous challenges adapting to independent life due to the demands of diabetes management. When adolescents change from dependence to independence they are not always prepared for what life will bring them, and when the adolescent has a chronic condition like Type 1 diabetes, the challenges are magnified. Maintaining diabetes management behaviors can be challenging for young adults.

Students With Diabetes is a national campus-based education and peer support program for young adults with diabetes. The program is designed to help young adults better cope by connecting young adults to provide peer social support and diabetes education. The program is based on the premise that perceived social support improves disease management and quality of life. Currently there are 26 college chapters across the United States. The objectives of the program are to empower young adults living with diabetes, to socially connect young adults with diabetes, to create a social safety net for young adults with diabetes and to provide convenient, relevant, socially acceptable diabetes education targeted to the young adult life stage.

An evaluation of SWD was conducted to assess the utility of diabetes education and social support, and effects on diabetes control measures. Participants completed surveys and standardized HbA1c blood glucose measures at the end of each semester. The assessment measures perceived social support, benefit from SWD membership and diabetes management outcomes.

Overall, respondents' $\mathrm{HbA1c}$ measurements improved over the academic year. This trend reiterates the increased motivation students reported to maintain a healthier lifestyle.The results indicate that improved perceived support of young adults with diabetes can positively impact the individual's ability to manage lifestyle and diabetes needs. Further research is warranted. This model for health communication may be useful for health and chronic disease education on college campuses during the young adult transition phase.

Supported by: The Patterson Foundation

2463-PO

The Assessment of Baseline Self-Management, Self-Care Ability and Medication Adherence of Enrolled Patients In OPENING (Organization Program of DiabEtes INsulIN ManaGement) Study JIE LIU, LINONG JI, JUMING LU, XIAOHUI GUO, Taiyuan, China, Beijing, China

Purpose: To evaluate the patients' ability of self-management, self-care and medication adherence at baseline.
Methods: T2DM patients over 18 years and inadequately responded to two or more oral anti-diabetic treatments (OADs) for over 3 months ( $\mathrm{HbA} 1 \mathrm{c} \%$ $>7.5 \%$ ). Patients were randomized (1:1) into the education group (structured education plus insulin therapy) and the control group (usual care plus insulin therapy). Chinese version Diabetes Management Self-efficacy Scale (C-DMSES), Summary of Diabetes Self-Care Activities Scale (SDSCA), and Morisky Medication Adherence Scale (MMAS) were used to assess selfmanagement, self-care and medication adherence. Baseline scores of these scales rated by patients were collected and assessed.

Results: Totally 1511 patients with T2DM were enrolled from 48 hospitals across China. The total score of C-DMSES is $153.34 \pm 34.43$ (full score 200). Patients rated higher scores in taking medicines on time according to physicians' prescription, maintaining anti-diabetic medication during illness, and self-monitoring blood glucose; meanwhile rated lower scores in following a healthy diet plan during special holiday, on party or dining out. The total score of SDSCA is $54.95 \pm 14.10$ (full score 84). Patients rated higher scores in following physicians' prescription to take anti-diabetic drugs, keeping feet dry and wearing comfortable shoes; meanwhile rated lower scores in controlling high-fat food intake and self-monitoring blood glucose. The proportion of patients with medium MMAS score and low MMAS score were $40.56 \%$ and $17.37 \%$, respectively. About $42.81 \%$ patients had ever forgotten to take medications; $27.95 \%$ sometimes paid no attention to medicines; $24.24 \%$ and $20.07 \%$ had withdrawn medicines.

Conclusions: Over $40 \%$ of patients forgot or withdrew medications, and nearly $30 \%$ of patients paid no attention to take medicines.

\section{Advancing the Scope of Diabetes Educators}

\section{JACKIE BOUCHER, GRETCHEN BENSON, Minneapolis, MN}

Despite national guidelines to treat patients diagnosed with chronic conditions, challenges remain in treating individuals to recommended targets. One approach to achieve clinical goals in these patients seeks to expand the scope of practice for traditionally non-prescribing healthcare professionals. We conducted a systematic review to examine the evidence to support the role of dietitians, nurses and pharmacists in the initiation or adjustment of pharmacotherapy to manage diabetes, dyslipidemia, and hypertension using physician-approved protocols or treatment algorithms

Figure 1 describes the methods used to identify and select the 19 articles. Medication management using physician-approved protocols can lead to clinically significant improvements in diabetes, dyslipidemia, and hypertension. (Table 1). A collaborative, multidisciplinary approach can help to ensure patients receive timely intensification of therapeutic medications and needed coaching for lifestyle change.

\section{Systematic Review Outcomes}

\begin{tabular}{llll}
\hline Health Authors, Year & Chronic & Notable outcomes \\
Professional $\&$ Study Type & Condition & \\
\hline
\end{tabular}

Dietitian 1.Robinson, Conroy, \& Hyperlipidemia, 1.The proportion of secondary preWickmeyer, 2000 (case Dyslipidemia vention patients with LDL cholesterol series)

2.Worth \& Davies, 2006

(time series)

$<100 \mathrm{mg} / \mathrm{dL}$ had almost doubled ( 1 vs. $32 \%$ p $<0011$ 2 Total cholesterol fell from a mean of $241(95 \% \mathrm{Cl}$ $235-247)$ to $169 \mathrm{mg} / \mathrm{dL}(95 \% \mathrm{Cl} 166-$ 172). Triglycerides fell from $268195 \%$ Cl 229-307) to $184 \mathrm{mg} / \mathrm{dL}(95 \% \mathrm{Cl}$ $166-203)(p<.001)$

\begin{tabular}{lll}
\hline Nurse & $\begin{array}{l}\text { 1. Andersen, Simper, } \\
\text { lbsen, \& Svensen, 2010 }\end{array}$ & $\begin{array}{l}\text { Hypertension } \\
\text { (3); Hyperlipi- } \\
\text { (prospective cohort) }\end{array}$ \\
demia and \\
2.New, et al., 2003 (RCT) & hypertension (1) \\
3. Rudd et al., 2004 (RCT) & \\
4. Tobe et al., 2006 (RCT)
\end{tabular}

1.Mean systolic and diastolic blood pressure dropped $34 \mathrm{mmHg}$ and 15 mmHg, respectively. 2. The intervention group achieved goals for blood pressure and/or hypertension more often than control subjects $(p=.003)$ 3. The intervention group's systolic blood pressure fell by $14.2 \mathrm{~mm} \mathrm{Hg}$ $(95 \%$ Cl -18.1 to -10.0$)$ compared to $5.7 \mathrm{~mm} \mathrm{Hg}$ in the usual care group at 6 months $(95 \%$ Cl -10.2 to -1.3$)$ ( $p<$ 01). 4. Diastolic blood pressure reductions were $4.8 \mathrm{~mm} \mathrm{Hg}$ greater in the intervention group compared to the control group ( $p=.05$ ). No statistically significant change in systolic blood pressure between groups. 


\begin{tabular}{|c|c|c|c|}
\hline $\begin{array}{l}\text { Dietitian, } \\
\text { nurse }\end{array}$ & $\begin{array}{l}\text { 1. Senior, MacNair, } \\
\text { \& Jindal, } 2008 \\
\text { (prospective cohort) }\end{array}$ & $\begin{array}{l}\text { Diabetes, } \\
\text { dyslpidemia, } \\
\text { hypertension }\end{array}$ & $\begin{array}{l}\text { Systolic blood pressure: } 133 \pm 19.3 \\
\text { to } 129 \pm 16.6 \mathrm{~mm} \text { Hg; diastolic blood } \\
\text { pressure: } 74+11.3 \text { vs. } 71 \pm 9.7 \mathrm{~mm} \\
\text { Hg; A1C: } 8.1+1.9 \text { vs. } 7.5+1.3 \% \text {; } \\
\text { LDL cholesterol: } 104+35 \text { vs. } 93+31 \\
\mathrm{mg} / \mathrm{dL}, p<.0001 \text { for all). }\end{array}$ \\
\hline \multirow[t]{14}{*}{ Pharmacist } & 1.Anaya et al., 2008 & \multirow{3}{*}{$\begin{array}{l}\text { Diabetes (2); } \\
\text { Diabetes \& } \\
\text { Dysplipidemia } \\
\text { (2) }\end{array}$} & \multirow{14}{*}{ 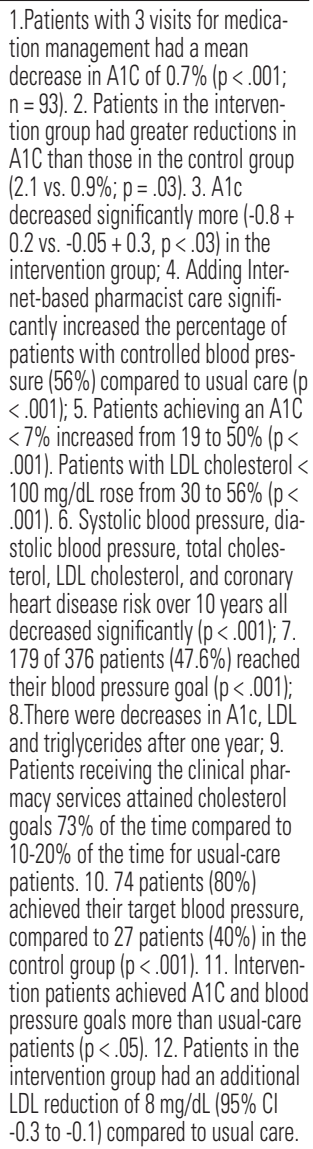 } \\
\hline & $\begin{array}{l}\text { (retrospective cohort) } \\
\text { 2. Choe et al., } 2005 \text { (RCT) }\end{array}$ & & \\
\hline & 3. Davidson Karlan, \& & & \\
\hline & Hair, 2000 (Case-control) & Hypertension (5) & \\
\hline & $\begin{array}{l}\text { 4. Green et al., } 2008 \\
\text { (RCT) }\end{array}$ & \multirow{10}{*}{$\begin{array}{l}\text { Diabetes, } \\
\text { dyslipidemia \& } \\
\text { hypertension (1) } \\
\text { Dyslipidemia (2) }\end{array}$} & \\
\hline & 5. Kiel \& McCord, 2005 & & \\
\hline & & & \\
\hline & $\begin{array}{l}\text { 6. Lowey et al., } 2007 \\
\text { (Prospective cohort) }\end{array}$ & & \\
\hline & $\begin{array}{l}\text { 7. McConnell et al., } 2006 \\
\text { (Prospective cohort) }\end{array}$ & & \\
\hline & $\begin{array}{l}\text { 8. Morello et al., } 2006 \\
\text { (retrospective cohort) }\end{array}$ & & \\
\hline & $\begin{array}{l}\text { 9. O'Donnell, Chen, } \\
\text { \& Piziak, } 2001 \\
\text { (retrospective cohort) }\end{array}$ & & \\
\hline & $\begin{array}{l}\text { 10. Reid, Murray, \& } \\
\text { Storrie, } 2005 \text { (RCT) }\end{array}$ & & \\
\hline & $\begin{array}{l}\text { 11. Taveira et al., } 2010 \\
\text { (RCT) }\end{array}$ & & \\
\hline & $\begin{array}{l}\text { 12. Villeneuve et al., } \\
2010 \text { (RCT) }\end{array}$ & & \\
\hline
\end{tabular}

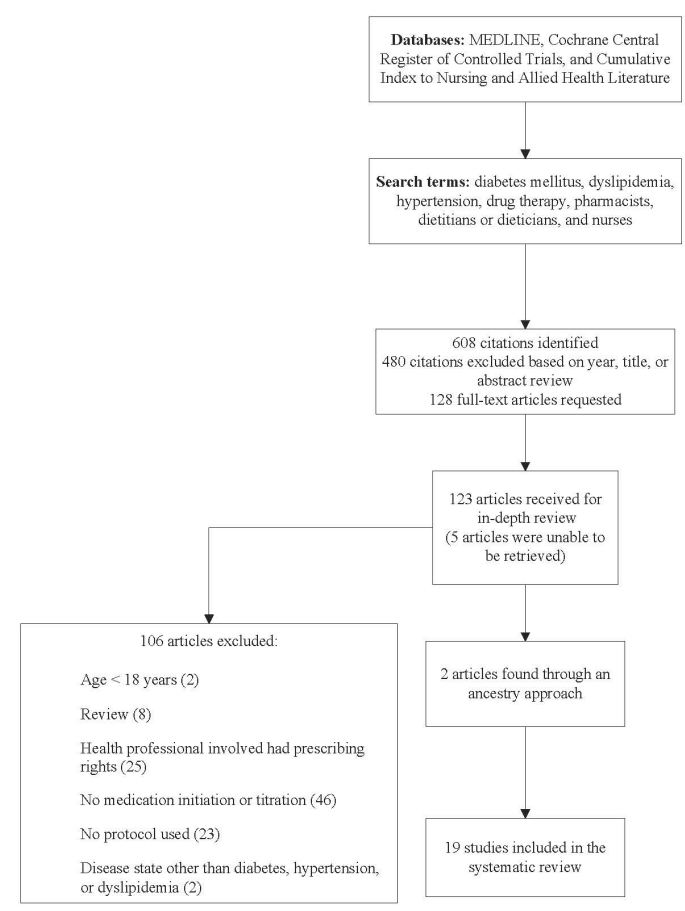

Figure 1. Flow chart of literature search strategy, review process, and results. Of 608 identified citations, 19 were selected for inclusion in the systematic review.
2465-PO

Addressing the Burden of Diabetes Among American Indian Youth RACHEL STROM, NICOLE NEAULT, GERILENE TSOSIE, ROCHELLE LACAPA, BRITTA MULLANY, KRISTIN LAKE, RAYMOND REID, ALLISON BARLOW, Baltimore, MD

American Indian youth have the highest and fastest growing rate of type 2 diabetes of any ethnic group in the US and face cultural and structural barriers to treatment and prevention.1 Our purpose is to present the design and methods of the Johns Hopkins Center for American Indian Health Together on Diabetes pilot trial conducted in partnership with four Southwestern American Indian communities to promote healthy lifestyle changes and health care coordination for youth ages 10-19 with or at risk for type 2 diabetes. A Community Based Participatory Research (CBPR) process, including a community stakeholder-led gaps and assets analysis, shaped the following design of the trial: Adolescents $(n=250)$ and an affiliated "support person" (primary guardian or selected surrogate) are recruited to receive a 12-session homevisiting intervention administered by American Indian paraprofessional "Family Health Coaches." The curriculum was adapted from the Diabetes Prevention Program (DPP), an evidence-based program with documented impact in adult populations including American Indians, but not youth.2-4 Four key adaptations driven by participating populations were: 1) increased focus on holistic wellness vs. weight loss; 2) a computer-based culturally relevant animated story series to reinforce key content; 3 ) a computerized system to measure and reward incremental knowledge and behavior change; and 4 Family Health Coach-assisted visits to community clinical providers to improve treatment compliance and care coordination. A mixed methods assessment administered at four intervals (baseline, 3, 6 and 12 months) measures intervention impact on knowledge, dietary intake, physical activity, emotional well-being, physiological change, and family and system change. If study aims are achieved, the public health field will have preliminary evidence for an innovative community health worker-centered model to avert the negative consequences of type 2 diabetes for American Indian youth and families.

Supported by: Bristol-Myers Squibb

\section{Myths on Diabetic Diet in Central European Population}

2466-P0

MALGORZATA U. NAPIERALA, DOROTA HERMANN, KATARZYNA HOMA, MARTA E. BRYSKIEWICZ, IZABELA GUTOWSKA, LILIANNA MAJKOWSKA, Police, Poland

Among diabetic patients, there are many myths concerning food products which are believed to lower or - at least - not influence the glucose level, even though they contain carbohydrates. The aim of this study was to assess the knowledge of diabetic patients and hospital nurses concerning popular food products and their impact on blood glucose levels.

The study group consisted of 250 patients with diabetes (DM), members of the Polish Diabetes Association; the control group (C) consisted of 123 healthy nurses from three hospitals in Szczecin, Poland. Participants were asked to complete a questionnaire on products common in diabetic diet and their influence on blood glucose levels. The products were: grapefruit, honey, wheat coffee, diabetic chocolate, milk soup with flakes and pork chuck steak. The highest percentage of wrong answers was given for pork (DM 65\%; C $74 \%, p<0,01$ ) and grapefruit (DM 51\%; C 77\%, $p<0,01$ ), while the most correct answers were for honey (DM 69\%; C 80\%, p<0,05) and milk soup (DM 64\%; C $68 \%$, NS). Negative correlation was found between the number of correct answers and the age of patients $(r s=-0,14, p<0,01 ;)$; while, there was no correlation between the number of correct answers and the duration of diabetes mellitus (NS).

1. The level of knowledge concerning products commonly used in diabetic diet among patients with diabetes and hospital nurses is low. 2. Both groups, patients and hospital nurses, need education as to the diabetic diet.

\section{7-PO}

Cities for Life: Community-Wide Resources for Diabetes Management in Birmingham

EDWIN P. FISHER, NATALIA LOSKUTOVA, LISAM. JONES, JOANICEA. THOMPSON, ANDREA L. CHERRINGTON, T. MICHAEL HARRINGTON, TAMELA J. TURNER, WILSON D. PACE, Chapel Hill, NC, Leawood, KS, Birmingham, AL, Aurora, CO

American Diabetes Association and American Association of Diabetes Educators guidelines call for diabetes self-management support to sustain the benefits of self management education during the 8,760 hours a year individuals are "on your own" to manage diabetes. Multi-level clinical, policy and health promotion campaigns such as those which have reduced smoking in the US and cardiovascular risks in Finland point to the potential of broad community approaches to self management support. Cities for Life in Birmingham, Alabama combines support from the city mayor and key councilors, primary care providers, patient navigators, voluntary health organizations 


\section{EXERCISE-ANIMAL}

that provide a variety of management and lifestyle programs, the Department of Family Medicine and Division of Preventive Medicine of the University of Alabama at Birmingham, broad social marketing, and primary care and health promotion expertise of the American Academy of Family Physicians Foundation, all coordinated through a broadly representative Community Action Team and a Steering Committee of four lead organizations. Success to date includes recruitment of over 70 organizations to the Community Action Team, participation in 19 local organizations' events and meetings reaching more than 6,640 people, 2 city-wide events with extensive earned print, broadcast and digital media coverage that gained over 5.5 million media impressions, deployment of patient navigators linking primary care providers and their patients with local resources, and development of a local website to help individuals and navigators access resources. New linkages developed between organizations include that between the YMCA and the university's HealthSmart program to coordinate lifestyle and health promotion services. Further growth will focus on collaborations among Community Action Team organizations to enhance community resources for diabetes management, and gaining business, health system and government support to sustain the program.

Supported by: Sanofi U.S.

2468-P0

Diabetes Self-Management Score System Implemented in 8 Public Hospitals, Botswana

VERONIQUE LA HAUSSE DE LALOUVIERE, Quatre Bornes, Mauritius

In 2010, a diabetes education program was initiated by APSA International (NGO) in collaboration with MOH Botswana. To date, 146 HCP's (98 nurses, 22 doctors, 28 paramedical staff), from 6 district and 2 referral hospitals, have been trained in patient diabetes education. Consequently 2143 patients have been enrolled in this educational network. The purpose is to empower people with diabetes to reduce their risk factors by adopting healthy life style behaviours, using a booklet "Learning to Control Cardiovascular Risks." This booklet was developed by Balcou-Debussche (2006), adapted and translated into Setswana. During the group session, each participant's perception about his disease is confronted with evidence-based knowledge, relating to the health of his arteries. The participant is lead to decide which activities (e.g. walking, quit smoking, etc...) are most suitable for him. Colour Codes and local pictures help participants with low literacy. Their variables, such as blood pressure, blood glucose, lipids profile, waist circumference, etc, are recorded. Each variable has a corresponding score value (for instance the score is 0 if systolic is $\geq 180 \mathrm{mmHg}$ and 10 if systolic is $\leq 130 \mathrm{mmHg}$ ). The participant will write his scores next to each risk factor and work out his "Health Total" in relation to a maximum score of 60 . His choice of activities is motivated by his desire to increase his Health Total (e.g. by choosing to reduce salt intake, 2 points are added to his Blood Pressure score). The participants will attend a follow up session every 6-9 months. The recorded variables in the booklet enable evaluation of risk factor 'improvement. The way forward would be to compare variables collected on specific groups of patients. An evaluation of results from a similar education program in Burundi has shown significant risk factor improvement. In conclusion, APSA's trainings have shown to be a catalyst in promoting low cost and efficient patient self-management in Botswana.

Supported by: World Diabetes Foundation

\section{EXERCISE-ANIMAL}

\section{9-P0}

The Role of VEGFR1TK Signaling in Blood Flow Recovery in Streptozotocin Induced Diabetic Mice

KAZUHITO OBA, HIDEKI AMANO, TAKEHITO SATO, FUMIHIRO OGAWA, SHINICHIRO OKIZAKI, TATSUMI MORIYA, MASAYOSHI SHICHIRI, MASATAKAMAJIMA, Sagamihara, Japan

It has been well known that diabetes mellitus (DM) is associated with endothelial cell dysfunction and reduced neovascularization in response to ischemic condition. Vascular endothelial growth factor (VEGF) induces angiogenesis through two tyrosine kinase receptors, VEGFR1 and VEGFR2. But the precise mechanism of VEGF receptors signaling in DM is not well understood. We hypothesized whether the expression of VEGF receptors signaling in DM mice impaired from recovery from ischemic condition or not. (Methods) 6-8 weeks C57BI/6 mice (WT) and VEGFR1-tyrosine kinase knockout mice (VEGFR1TKKO) were used. Diabetes was induced by a single intraperitoneal injection of $100 \mathrm{mg} / \mathrm{kg}$ streptozotocin (STZ). The model of hind limb ischemia was made by ligated the femoral artery in the right limb. Angiogenesis was estimated by measuring blood flow ratio by using Laser Doppler. The expression of VEGF receptorwas analyzed by real time PCR analysis. Furthermore, VEGFR1+CXCR4+,

\section{EXERCISE-HUMAN}

bone marrow derived cell were detected by flow cytometry and immunofluorescent analysis.

(Results) Compared to control mice, blood flow recovery was significantly suppressed in STZ induced DM mice (Control 0.70 \pm 0.05 , DM 0.37 \pm 0.06 $P<0.05)$. The expression of VEGFR1 was significantly suppressed in DM mice compared to VEGFR2 $(P<0.05)$. There was no significant change in the number progenitor cells, VEGFR1+CXCR4+ cells, between DM and control mice but the accumulation of VEGFR1+CXCR4+ cells were significantly suppressed in DM ischemic muscle.

Furthermore, there was no significant change in blood recovery between VEGFR1TKKO mice $(0.48 \pm 0.15)$ and STZ treated VEGFR1TKKO mice $(0.45 \pm 0.28)$. These results suggested that DM suppress blood flow recovery from ischemic condition by down regulation of VEGFR1TK signaling. Control the regulation of VEGFR1TK signaling might be a new tool for recovering from ischemic condition.

\section{WITHDRAWN}

2470-P0

\section{EXERCISE-HUMAN}

\section{1-P0}

WITHDRAWN 
2472-PO

Effects of Moderate Physical Activity on Metabolic Control in Type 1 Diabetes: A 3-Years Experience

ROBERTO ANICHINI, PIERGIORGIO FRANCIA, NICOLA MOCHI, ANNA TEDESCHI, SANDRO ANICHINI, MASSIMO GULISANO, ALESSANDRA DE BELLIS, Pistoia, Italy, Firenze, Italy

Exercise is recommended in type 1 diabetes mellitus (DM1), but longstanding effects of moderate physical activity (MPA) on metabolic control is discussed.

Aim of this study was to evaluate $\mathrm{HbA} 1 \mathrm{c}$ and cardiorespiratory performance (CP) after a MPA in sedentary subjects with DM1 in the 3 years following the training protocol.

In 2009 in a group of 14 sedentary DM1, only $8(5 / 3 \mathrm{M} / \mathrm{F})$, mean age $36,5 \pm$ $10,6 \mathrm{yr}$, duration of diabetes $17,4 \pm 8,6 \mathrm{yr}$, mean $\mathrm{HbA1c} 8,0 \pm 1,6 \%$ concluded the training period.

$\mathrm{HbA1c}$ and $\mathrm{CP}$ were evaluated before and after 4 months of MPA. HbA1c was evaluated 3 years after MPA.

All subjects followed a training protocol 4 times a week for 16 weeks

Plasma glucose level was checked 2 hours before training, at the beginning, after 30 minutes and at the end of MPA and before going to bed.

The training program consisted in 10 minutes organic activation, 30 minutes MPA at $45-65 \%$ of V02 peak, and 10 minutes postural exercise and stretching.

At the beginning of the study all subjects were given information about prevention and management of hypoglycaemia.

After 16 weeks of training HbA1c improved $(-0.87 \% \pm 0.18, p<0,1)$ and peak $\mathrm{V} 02 / \mathrm{Kg}$ was increased from 31,4 to $34,5 \mathrm{~mL} / \mathrm{kg} / \mathrm{min}(3.1 \pm 1.66, \mathrm{p}<0,01)$. Peak muscle power output improved from 174 to 194 watt $(20.16 \pm 16.85, p<0,05)$. Anaerobic threshold increased: V02/Kg from 17.3 to $19.9 \mathrm{~mL} / \mathrm{kg} / \mathrm{min}(2.6 \pm$ $2.43, p<0,05)$ and peak aerobic power output from 82.5 to 97.5 watt $(15,0 \pm$ $9.35, \mathrm{p}<0,01)$.

After 3 years from the beginning of MPA HbA1c improved in comparison with values detected before the training period: $0.81 \% \pm 0.9, P<0.05$.

The sedentary subjects with DM1 are able to perform MPA and improve since the early months their cardiorespiratory condition and metabolic control. It is noteworthy that these effects seem to last until 3 years from the beginning of the protocol, probably in relation to an incisive change of lifestyle.

The prevention of the hypoglycaemia is linked to correct information of patients about possible adjustment of insulin therapy or nutritional intake.

\section{3-P0}

Caffeine HasMinimal Impact on Interstitial Blood Glucose Concentrations during Exercise in Individuals With Type 1 Diabetes: A Preliminary Study

DESSI P. ZAHARIEVA, VERONICA JAMNIK, MICHAEL C. RIDDELL, Toronto, ON, Canada

Although regular exercise is considered beneficial for individuals with type 1 diabetes (T1D), it is frequently associated with hypoglycemia, both during activity and in recovery. Although caffeine has been used as an ergogenic aid for exercise and sport in healthy, non-diabetic individuals, no studies to date have been conducted to examine the effects of caffeine on glycemia, or performance, during exercise specifically in individuals with T1D. The purpose of this study was to determine the effects of caffeine ingestion on glycemia during steady state moderate-intensity aerobic exercise. Eight healthy subjects (age $25.3 \pm 9.0$ (years $\pm S D$ ), weight $71.9 \pm 13.5 \mathrm{~kg}$, height $172.1 \pm 12 \mathrm{~cm}$, V02max) $43.4 \pm 12.7 \mathrm{~mL} / \mathrm{kg} / \mathrm{min}$, body fat $20.6 \pm 8.8 \%$, waist circumference $82.9 \pm 5.4 \mathrm{~cm}$, duration of diabetes $12 \pm 9$ years, HbA1c $7.4 \pm 0.8 \%$ ) with T1D on insulin pump therapy (62.5\%) or multiple daily injections (37.5\%) that are regular caffeine users were recruited for the study. Subjects ingested capsules that contained $6.0 \mathrm{mg} / \mathrm{kg}$ of body weight of either gelatin (placebo) or pure caffeine powder. Subjects exercised for 45-minutes in the late afternoon (1600h) on a treadmill at 60\% V02max on two separate visits (caffeine and placebo) with only circulating basal insulin levels. Capillary blood glucose was measured at 0-, 5-, 15-, 30-, and 45-minutes during exercise. Slight elevations in blood glucose levels were noticed during the caffeine trials at 15-minutes into exercise $(p=0.1)$ in comparison to the placebo trials, although this difference was not statistically significant. A paired t-test determined no difference in rate of perceived exertion $(p=0.8)$, heart rate $(p=1.0)$, or V02 $(p=0.1)$ between trials. We conclude that caffeine ingestion 1 -hour prior to the onset of exercise has no effect on blood glucose concentrations and does not reduce the likelihood of hypoglycemia in comparison to no caffeine.
2474-PO

Health Supported Exercise Intervention in Patients With Metabolic Syndrome: A Randomized Clinical Trial MELANIE I. STUCKEY, DAWN P. GILL, KRISTIN J. SABOURIN, SHEREE SHAPIRO, REBECCA A. HUBBARD, ROBERT J. PETRELLA, London, ON, Canada, Seattle, WA

The purpose was to determine whether an interactive mHealth exercise intervention was more effective than standard of care exercise in patients with metabolic syndrome. Participants [n=127, mean age 57.4 (SD 9.1) y, 74\% female] reported to the laboratory at baseline (V0) and follow-up [12 (V1), 24 (V2), and 52 (V3) weeks]. Anthropometrics and blood pressure (BP) were measured and blood drawn to examine fasting glucose (FG), glycated hemoglobin $(\mathrm{HbA1C})$, lipids, insulin and high sensitivity C-reactive protein (CRPhs). Fitness (V02max) was assessed and individualized exercise programs were pre scribed. The intervention group received a Smartphone data portal and Bluetooth ${ }^{\text {TM }}$ enabled devices for biometric tracking. Differences between groups in outcomes (V1-V0) were examined using analyses of covariance, which adjusted for baseline levels of the outcome of interest. Group differences from V0 to V3 were examined with two-way repeated measures ANOVAs. At $\mathrm{V} 1$, systolic BP was reduced in both groups but significantly more in the control group (difference in mean change: $-5.68 ; 95 \% \mathrm{Cl}:-10.86,-0.50, p=0.03$ ) and CRPhs was also significantly reduced in the control group (difference in mean change: $-0.45 ; 95 \% \mathrm{Cl}:-0.88,-0.01, p=0.04)$. There were no differences between groups at V1 for other outcomes. Across the follow-up period, systolic BP, diastolic BP, waist circumference, total cholesterol, LDL and HbA1c were decreased, and V02max was increased for the entire study population $(p<0.001)$, with no difference in rate of change between groups. There were no changes in triglycerides or HDL. FG, insulin and CRPhs were significantly higher in the intervention group, compared to the control group, across the entire follow-up period $(\mathrm{p}<0.05)$ and $F G$ increased over time for the entire study population $(p=0.01)$. Over 52 weeks, cardio-metabolic risk factors improved with both standard and mHealth supported exercise interventions.

2475-PO

Eccentric Endurance Exercise Significantly Lowers Liver Enzymes in Overweight and Obese Individuals

HEINZ DREXEL, PHILIPP REIN, ALEXANDER VONBANK, HELGA WINKLER, CHRIS-

TOPH H. SAELY, Philadelphia, PA, Feldkirch, Austria, Triesen, Liechtenstein

Elevated liver enzymes are highly prevalent in overweight and obese patients, reflect the presence of non-alcoholic fatty liver disease, and are associated with an increased risk of diabetes and cardiovascular events. Liver enzymes can be lowered by physical exercise, but many overweight patients are not willing or not able to engage in strenuous exercise regimens. Eccentric endurance exercise (e.g. hiking downwards) is less strenuous than concentric exercise (e.g. hiking upwards) but its effects on liver enzymes are unknown.

We allocated 42 overweight and obese sedentary individuals to an exercise intervention program, consisting of hiking downwards a pre-defined route in the Austrian Alps over two months. For the opposite way, a cable car was used where compliance was recorded electronically. The difference in altitude was 540 metres; the distance was covered three to five times a week. A matched group of 12 individuals served as a control group. Metabolic profiles were obtained at baseline and after the two months period.

Compared to baseline, 8 weeks of eccentric endurance exercise significantly lowered serum alanine aminotransferase ( $\mathrm{ALT} ; 36 \pm 23$ vs. $31 \pm 18 \mathrm{U} / \mathrm{l} ; \mathrm{p}<0.001$ ), the ALT / aspartate aminotransferase (AST) ratio $(1.22 \pm 0.41$ vs. $1.02 \pm 0.33$; $\mathrm{p}<0.001)$, and serum gamma-glutamyl transferase $(56 \pm 98 \mathrm{vs.} 44 \pm 65 \mathrm{U} / \mathrm{l}$; $p=0.005)$, whereas these parameters did not change significantly in the control group ( $p=0.261, p=0.272$, and $p=0.644$, respectively). Eccentric enddurance exercise was well tolerated and there were no series adverse events.

We conclude that eccentric exercise is a promising new exercise modality which significantly lowers liver enzymes in overweight and obese individuals and therefore is of interest as a therapeutic intervention in non-alcoholic fatty liver disease patients. 
How to Reduce the Risk of Hypoglycaemia in Case of Physical A ity in Patients With Type 1 Diabetes Under Pump Therapy?

SYLVIA FRANC, MARIE-HÉLĖNE PETIT, ODILE JUY, AHMED DAOUDI, LUDOVIC BOURSIQUOT, CATHERINE PETIT, ARMELLE POCHAT, PHILIPPE DUPONT, LYDIE CANIPEL, FRANÇOIS KOUKOUI, GUILLAUME CHARPENTIER, Corbeil-Essonnes, France, Evry, France

In T1 diabetic (T1D) patients under pump therapy, recommendations are lacking regarding the adjustment of insulin doses in case of physical activity (PA). Our objective was to test several adjustments of insulin doses, taking into account intensity, duration of PA and time relative to meals.

Twenty T1D patients practicing leisure PA with an $\mathrm{HbA} 1 \mathrm{C}<9 \%$, were included. They had a $\mathrm{V}_{\max }$ determination and then performed 4 physical tests at least 48 hours apart (30-min cycling sessions on an ergonomic bicycle) $3 \mathrm{~h}$ after lunch, in a random order, at 2 levels of PA (moderate and intensive, i.e. $50 \%$ and $75 \% \mathrm{VO}_{\max }$ ) and with 2 options regarding the basal rate (BR): BR reduced by $50 \%$ or $80 \%$ in case of $50 \% \mathrm{VO}_{\max }$ and by $80 \%$ or $100 \%$ in case of $75 \%$ VO2 $2_{\text {max. }}$. BR was reduced during each exercise period $+2 \mathrm{hrs}$ to prevent hypoglycemia (except for the last one when pump was switched ON at the end of exercise). A rest test served as a reference. Blood glucose (BG) values were measured during the exercise (or rest) period and the following $2 \mathrm{hrs}$. Two additional tests at $50 \% \mathrm{VO}_{\max }$ were performed, $90 \mathrm{~min}$ after lunch to compare basal rate $v$ s. bolus reduction. We focused on the evolution of $B G$ values during the exercise period $+2 \mathrm{hrs}$.

Patients (11 men, BMI $24 \pm 3 \mathrm{~kg} / \mathrm{m} 2$, age $45 \pm 12 \mathrm{yrs}$, HbA1c at baseline $7.9 \%$, diabetes duration $18 \pm 9$ yrs, under pump therapy for $5 \mathrm{yrs}$ ) used to practice 4h/week of PA. Between 09/2011 and 12/2012, 113 physical tests out of 140 were performed. In all cases, the nadir of $B G$ values occurred at the end of the exercise session: for both $50 \% \mathrm{VO} 2_{\max }$ sessions, the reduction in $\mathrm{BG}$ was $-4 \mathrm{mM}$ vs $-0.2 \mathrm{mM}$ for rest test; for $75 \% \mathrm{VO2}_{\max }$ sessions, BG reduction was rather similar ( $\triangle \mathrm{BG}-3.7$ and -4.0 for BR-100\% and BR- $80 \%$ vs $-0.2 \mathrm{mM}$ ). Mean $B G$ values during the 2 following hrs then remained in an adequate range.

These results highlight the predominant action of insulin on board and they should lead health care providers to advise patients to reduce BR at least half an hour before the onset of exercise to prevent the occurrence of hypoglycemia.

Supported by: EFSD

\section{8-P0}

Eccentric Endurance Exercise Significantly Improves Both Fasting and Postchallenge Metabolism in Overweight and Obese Individuals

PHILIPP REIN, CHRISTOPH H. SAELY, ALEXANDER VONBANK, SIMONE GELLERRHOMBERG, HEINZ DREXEL, Feldkirch, Austria, Triesen, Liechtenstein, Philadelphia, $P A$

Eccentric endurance exercise (e.g. hiking downwards) is less strenuous than concentric exercise (e.g. hiking upwards) but data on its potential to reduce cardiovascular risk are scarce.

We allocated 68 overweight and obese sedentary individuals to an exercise intervention program, consisting of hiking downwards the same route over two months. For the opposite way, a cable car was used where compliance was recorded electronically. The difference in altitude was 540 meters; the distance was covered three to five times a week. A matched group of 12 individuals served as a control group. Fasting and postprandial metabolic profiles were obtained at baseline and after the two months period.

Compared with baseline, eccentric endurance exercise significantly lowered fasting glucose $(99 \pm 17$ vs. $96 \pm 13 \mathrm{mg} / \mathrm{dl} ; \mathrm{p}=0.036)$ as well as glucose tolerance following the oral intake of $75 \mathrm{~g}$ glucose $\left(250 \pm 49 \mathrm{vs}\right.$. $228 \pm 54 \mathrm{mg}^{*} \mathrm{dl}^{-1}$ $h ; p<0.001)$, whereas these parameters remained unchanged in the control group ( $p=0.495$ and $p=0.182$, respectively). Furthermore, eccentric endurance exercise significantly improved triglyceride tolerance in a standardized oral fat challenge test $\left(2121 \pm 1398\right.$ vs. $\left.1744 \pm 1143 \mathrm{mg}^{*} \mathrm{dl}^{-1} \mathrm{~h} ; \mathrm{p}<0.001\right)$, whereas triglyceride tolerance did not change significantly in the control group $(p=0.695)$. Body mass index was slightly but significantly lowered in the eccentric endurance exercise group ( $29.6 \pm 3.1 \mathrm{vs.} 29.2 \pm 3.3 \mathrm{~kg} / \mathrm{m}^{2} ; \mathrm{p}=0.004$ ) but not in the control group ( $p=0.237$ ).

Eccentric endurance exercise is a promising new exercise modality with favorable effects on both fasting and postchallenge metabolism.

2479-PO

Exercise Metabolic Fexibility in Association With Type 2 Diabetes SHINJI SATO, SHIGEKI TSUZUKU, SHINGO OTSUKI, SHIRO TANAKA, HIROYUKI KOSHIYAMA, Osaka, Japan, Kumamoto, Japan

Background: Metabolic flexibility, expressed as the change in respiratory quotient (RO) from the fasted state to the insulin-stimulated condition, indicates the insulin-sensitivity of muscles, possibly reflecting the ability of mitochondria to shift fuel selection (Diabetes, 2010). We have hypothesized that a delta $R 0$ during incremental exercise testing indicates metabolic flexibility and it decreases in patients with type 2 diabetes.

Purpose: We examined whether exercise metabolic flexibility is linked to development of type 2 diabetes.

Methods: In our study, 8 lean (L) and 20 obese (0) individuals without type 2 diabetes, and 8 obese individuals with type 2 diabetes (D), matched for age, underwent cardio pulmonary exercise testing (CPX). Exercise metabolic flexibility was defined as an increase in RO from the warming-up period to the maximal workload.

Results: Peak oxygen uptake was similar among the 3 groups $(L ; 19.5 \pm 4.0$

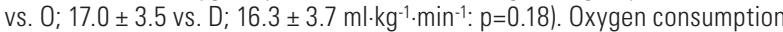
at anaerobic threshold (AT) was lower in groups 0 and $D$ than in group $L(L$; $14.4 \pm 2.4$ vs. $0 ; 11.3 \pm 2.2$ vs. $D ; 10.4 \pm 1.9 \mathrm{ml}^{\prime} \mathrm{kg}^{-1} \cdot \mathrm{min}^{-1}: \mathrm{p}=0.002$ ). Exercise metabolic flexibility was lower in group $D$ than in groups 0 and $L(L ; 0.21 \pm$ 0.06 vs. $0 ; 0.21 \pm 0.06$ vs. $D ; 0.14 \pm 0.07: p=0.03$ ).

Conclusions: The ability to shift substrate sources for energy use during exercise may be reduced in type 2 diabetic patients.

2480-P0

Increased Miles Walked Per Day Improves Proportion of Fat-Free Body Mass in Diabetics

HEATHER C. BRISLEN, GREGORY FOTIEO, CHRISTOPHER S. WENDEL, ELENA V. PLUMMER, JAYENDRA H. SHAH, GERALD A. CHARLTON, MARC T. MONTANARO, GLEN H. MURATA, Albuquerque, NM, Tucson, AZ, Phoenix, AZ

Among diabetics, increased physical activity is correlated with decreased mortality and cardiovascular disease. Data is mixed regarding the ability of podometer use to impact physical activity and weight loss goals, even though lifestyle activity seems as effective as structured exercise programs in improving activity levels and some health outcomes. Additionally, increasing proportion of fat mass is positively correlated with risk for chronic diseases, especially diabetes and cardiovascular disease, and percent fat-free body mass is likely to be a superior indicator of these risks than is BMI. In this cross sectional analysis we evaluated the validity of podometer data as a surrogate for activity level via correlation of fat free body mass to average miles per day by podometer. One hundred twenty-seven diabetic patients were recruited from a clinical trial of SMBG at the Albuquerque, Phoenix, and Tucson VA Medical Centers. The mean $( \pm$ SD) age was $64 \pm 7.4$ years, and $99 \%$ were male. Average BMI was $32.8 \pm 5.3 \mathrm{~kg} / \mathrm{m}^{2}$, and $\mathrm{HgbA1c}$ was $8.27 \pm 1.30$. Fat free body mass was assessed by bioelectrical impedance using a Bodystat Quadscan 4000 unit, and physical activity levels were tracked using seven consecutive days of podometer use as recorded by Yamax Digi-Walker podometers distributed at the outset of the study. Results demonstrated average fat free mass of $67.1 \pm 7.0 \%$ (range $40.7-83.4$ ), and average distance of $1.4 \pm$ 1.1 miles walked per day (range 0.03-5.92mi). Simple linear regression showed a positive correlation of miles per day with fat free mass (coefficient $2.71 \pm$ 0.54 , $r$-squared $=0.168 ; p<0.0000$.). For this population, one additional mile walked is likely to result in $2.71 \%$ improvement in fat free mass. In the average subject with weight of $221 \mathrm{lbs}$, this translates to 6.0 pounds lean body mass improvement. This effect remains significant when adjusting for age and gender.

Supported by: Roche Diagnostics Corporation 


\section{EXERCISE-HUMAN}

$\Delta$

2481-PO

Effect of Aging and Exercise Training on Lipomic Profiling in Human Subjects

HELEN LUM, LUIS ANAYA, JOSE DE JESUS GARDUNO GARCIA, RAWEEWAN

LERTWATTANARAK, NICOLAS MUSI, San Antonio, TX, Bangkok, Thailand

Aging is an important, independent risk factor for the development of insulin resistance and type 2 diabetes (T2DM), such that $~ 2 / 3$ of the older population has diabetes or impaired glucose tolerance (IGT). However, it is unclear why aging increases the risk of developing abnormalities in glucose metabolism. Since elevated free fatty acids (FFA) have been implicated in the pathogenesis of insulin resistance and $\beta$-cell dysfunction, we conducted lipomic profiling (C16:0, C16:1, C17:0, C18:0, C18:1, C18:2, C18:3, C22:4) of plasma samples from older lean normal glucose-tolerant (NGT; $n=14$, age $=65.9 \pm 3.1$ $y, \mathrm{BMl}=24.8 \pm 0.8 \mathrm{~kg} / \mathrm{m} 2, \mathrm{HbA} 1 \mathrm{c}=5.4 \pm 0.1 \%)$, older lean impaired glucose-tolerant (IGT; $\mathrm{n}=4$, age $=72.8 \pm 3.9 \mathrm{y}, \mathrm{BMI}=24.0 \pm 1.9 \mathrm{~kg} / \mathrm{m} 2, \mathrm{HbA} 1 \mathrm{c}=5.6 \pm 0.1 \%)$, and young lean NGT $(n=12$, age $=27.3+0.7 y, B M I=24.3+0.6 \mathrm{~kg} / \mathrm{m} 2, \mathrm{HbA1c}=5.1+0.1 \%)$ subjects. We also evaluated the effect of aerobic exercise on plasma FFA concentration. The average baseline concentration of all FFA was elevated in the older IGT as compared to older NGT (range $46-126 \%, P<0.05$ ). Two FFA species (C18:1, 67\%; C22:4, 52\%; $\mathrm{P}<0.05)$ were higher in older IGT versus young NGT subjects. Between NGT young and NGT older subjects, C18:3 and C22:4 were higher in the young $(34 \%, 52 \%, P<0.05$, respectively). The exercise program significantly decreased plasma C18:3 $(15 \%, \mathrm{P}<0.05)$ and C22:4 $(14 \%$ $\mathrm{P}<0.05$ ) concentrations, and tended to reduce (range $9-14 \%$ ) the other six analyzed FFA, in older IGT subjects. FFAs were not significantly altered by exercise in the younger NGT and older NGT groups. In summary, this lipomic profile reveals that older pre diabetic (IGT) subjects exhibit alterations in the plasma concentrations of several FFA, which might play a role in the pathogenesis of insulin resistance and $\beta$-cell dysfunction. Aerobic exercise reduced the concentration of most FFA, underscoring the value of physical activity in the prevention and treatment of T2DM.

Supported by: NIA; NIDDK

2482-PO

ELECTRODIAB Study: Impact of Bi-Quadricipital Muscular ElectroStimulation (MES) on Insulin Sensitivity (M-Value) in Type 2 Diabetic (T2D) Subjects

MICHAEL JOUBERT, LAURE METAYER, GAETAN PREVOST, ANNE ROD, JULIA MORERA, YVES REZNIK, Caen, France, Rouen, France

Physical activity improves insulin sensitivity and promotes type 2 diabetes (T2D) control. Muscular electro-stimulation (MES) enhances both muscles strength and volume. Metabolic effect of MES on insulin sensitivity is explored in this study.

12 patients $T 2 D$ were included lage $60,4 \pm 9,9$ years ; diabetes duration $12,9 \pm 7,5$ years; $\mathrm{BMI} 32,7 \pm 5,8 ; \mathrm{HbA1c} 7,2 \pm 0,8 \%$ ). Insulin sensitivity was evaluated with 3 euglycemic hyperinsulinemic clamps $\left(80 \mathrm{mUI} / \mathrm{m}^{2} / \mathrm{min}\right)$ for each participant : at baseline (BL) (TO), one hour after a unique MES session (T1), and after one week of daily MES training (T2). Each session of MES included a 25 minutes bi-quadricipital simultaneous stimulation with a 4 channel electrostimulator Compex ${ }^{\circledR}$ at maximal tolerated stimulation intensity. Subjects were asked to gradually increase stimulation intensity during the training week

For overall population (responders and non-responders), insulin sensitivity (M-Value) increased of $9,1 \pm 41,1 \%(p=0,68)$ and $30,6 \pm 40,3 \%(p=0,055)$ at T1 and T2 vs T0, respectively. For $5 / 12$ and $7 / 12$ responders, M-Value increased of $49,3 \pm 28,9 \%(p=0,062)$ and $58,0 \pm 29,1 \%(p=0,015)$ at T1 and T2 vs T0, respectively. During the first MES session, for a mean stimulation intensity of $26,2 \pm 6,1 / 100$, energy expenditure (EE) (indirect calorimetric measure) increased of $112,2 \pm 192,2 \mathrm{Kcal} / \mathrm{h}$ (ie $+6,1 \pm 10,2 \%$ from EE at rest) ( $p=0.065$ ) for overall population and of $200,8 \pm 88,1 \mathrm{Kcal} / \mathrm{h}(\mathrm{ie}+10,8 \pm 5,4 \%)(\mathrm{p}=0,009)$ for $9 / 12$ responders. During the daily MES training week, patients increased stimulation intensity with a week mean of $38,4 \pm 14,2 / 100$. Concerning tolerance, $8 / 12$ subjects felt moderate muscle pain $(n=7)$ and/or skin pain $(n=4)$ during or after MES sessions. All subjects completed the trial and 10/12 subjects required to use MES regularly after this trial.

This result suggests that further studies might explore MES as an alternate solution to physical activity in T2D.
2483-P0

Training to Run NY Marathon Promotes an Improvement in Metabolic Control for Fit Patients With Type 1 Diabetes

LUIS FORGA, LAURA CHINCHURRETA, MARÍA JOSÉ GOÑI, FRANCISCO JAVIER LAFITA, MARTA GARCÍA, JOSÉ MANUEL FERNÁNDEZ-REAL, Pamplona, Spain, Girona, Spain

Exercise offers important health benefits to patients with type 1 diabetes. Nevertheless, it has not consistently been shown to lead to improvement in glycaemic control as measured by HbA1c.

Our objectives have been evaluating changes in $\mathrm{HbA1c}$, fructosamine and other different parameters related with cardiovascular risk factors in patients with type 1 diabetes after eight months training to run the New York marathon.

We have followed 8 patients ( 7 men and 1 woman), measuring their blood pressure and body composition and analyzing their $\mathrm{HbA1c}$, fructosamine, lipid profile, PAl-1, leptin, irisin and adiponectin at the beginning of training period in February 2012 and at the end in October. All of them were under intensive treatment before the study, 6 with $\mathrm{MDI}$ and 2 with ISCI. The mean of their ages was 41.2 years (range: $22-49$ ). The mean of their diabetes duration was 13.5 years (SD: 6.82). Before the study period, they usually ran $35 \mathrm{Kms}$ three days every week, and during the study, they ran 70-80 Kms during five training days per week. Statistical analysis: We used the Wilcoxon test to evaluate differences between final and initial data.

The results are shown in Table 1.

Table 1. Basal values and changes after training

\begin{tabular}{|c|c|c|c|c|c|}
\hline & \multicolumn{2}{|c|}{ Pre-training value } & \multicolumn{2}{|c|}{ Post-training value } & \multirow{2}{*}{$\begin{array}{c}\begin{array}{c}\text { Statistical } \\
\text { difference }\end{array} \\
p\end{array}$} \\
\hline & Mean 1 & $\mathrm{Cl} 95 \% 1$ & Mean 2 & $\mathrm{Cl} 95 \% 2$ & \\
\hline HbA1c (\%) & 7.6125 & (6.88- 8.34) & 7.3875 & $(6.64-8.12)$ & 0.09 \\
\hline Fructosamine (umol/l) & 353.2500 & (302.27- 404.22) & 334.1250 & $(286.67-381.57)$ & 0.036 \\
\hline Weight (kg) & 74.90 & (67.76- 82.03) & 73.04 & $(66.48-79.54)$ & 0.176 \\
\hline Fat mass \% & 17.56 & (14.13-20.99) & 12.74 & $(8.54-16.94)$ & 0.018 \\
\hline $\mathrm{SBP}(\mathrm{mm} \mathrm{Hg})$ & 126.63 & (116.45-136.80) & 114.38 & (106.82-121.93) & 0.027 \\
\hline $\mathrm{DBP}(\mathrm{mm} \mathrm{Hg})$ & 71.13 & (64.27- 77.98) & 70.00 & (63.68- 76.32) & 0.785 \\
\hline Total Cholesterol (mg/dl) & 173.7500 & (144.52-202.97) & 172.0000 & $(147.86-196.13)$ & 0.889 \\
\hline $\mathrm{cLDL}(\mathrm{mg} / \mathrm{dl})$ & 97.3750 & (72.85- 121.89) & 91.0000 & (73.75- 108.24) & 0.231 \\
\hline $\mathrm{cHDL}(\mathrm{mg} / \mathrm{dl})$ & 65.6250 & (53.59- 77.65) & 71.3750 & $(58.50-84.24)$ & 0.050 \\
\hline Triglycerides (mg/dl) & 54.5000 & (42.96-66.03) & 48.3750 & (38.49-58.25) & 0.345 \\
\hline Leptin (ug/l) & 2.4125 & $(0.49-5.32)$ & 1.2375 & $(0.64-1.83)$ & 0.175 \\
\hline |risin (ng/ml) & 183.86 & (83.87-283.85) & 137.02 & (96.50-177.49) & 0.237 \\
\hline Adiponectin ( $\mu \mathrm{g} / \mathrm{ml})$ & 12.0875 & $(8.24-15.92)$ & 19.2375 & (11.29-27.17) & 0.012 \\
\hline PAl-1 (ng/ml) & 4.7625 & $(3.30-6,22)$ & 3.8125 & $(2.36-5.26)$ & 0.092 \\
\hline
\end{tabular}

Intensive training for athletic patients, under optimized insulin treatment trends to improve their glycaemic control. They also show an additional improvement in some cardiovascular risk factors, but leptin and irisin don't change.

Supported by: Fundación de Endocrinología; Nutrición y Diabetes de Navarra

2484-PO

\section{WITHDRAWN}




\section{WITHDRAWN}

2486-P0 Effects of Type 2 Diabetes on Perceived Difficulty of Exercise in Older Women

AMY G. HUEBSCHMANN, LEAH HERLACHE, WENDY M. KOHRT, JANE E.B. REUSCH, PAMELA WOLFE, JUDITH G. REGENSTEINER, Aurora, CO, Denver, CO

Premenopausal women with uncomplicated type 2 diabetes mellitus (T2D) perceive even low intensity exercise to require greater perceived exertion (RPE) than do overweight and normal-weight nondiabetic adults, possibly related to the impaired peak oxygen consumption ( $\mathrm{VO}_{2}$ peak) observed in $\mathrm{T} 2 \mathrm{D}$. Disproportionately greater RPE has been associated with reduced usual physical activity. To address whether RPE differs in postmenopausal women by T2D status, we measured RPE at low absolute ( 30 watts, W) and relative work intensities ( $35 \% \mathrm{VO}_{2}$ peak). We hypothesized RPE would be greater in women with T2D vs. no T2D. We enrolled overweight, sedentary women aged $50-75$ with $(n=26)$ and without $\mathrm{T} 2 \mathrm{D}(\mathrm{n}=28)$ and measured $\mathrm{VO}_{2}$ peak in all. Participants also performed 8-minute bouts of submaximal cycle ergometer exercise at $30 \mathrm{~W}$ ( 4 bouts) and at $35 \%$ of individual $\mathrm{VO}_{2}$ peak ( 4 bouts). We assessed RPE at both intensities with the Borg RPE scale (range 6-20, 6 = "no exertion at all", 20 = "maximal exertion") using a maximum likelihood repeated measures model. Groups were similar in age, $\mathrm{BMI}$, and usual physical activity levels. Although $\mathrm{VO}_{2}$ peak was lower in T2D (No diabetes: $17.8 \pm 3.0 \mathrm{ml} / \mathrm{kg} /$ $\mathrm{min}$, T2D: $15.4 \pm 2.5 \mathrm{ml} / \mathrm{kg} / \mathrm{min}, P=0.003)$, RPE in the $8^{\text {th }}$ minute of exercise was only non-significantly greater in T2D vs. controls (RPE 30W: No diabe- tes, $10.4 \pm 3.2, \mathrm{~T} 2 \mathrm{D}, 11.7 \pm 2.3, P=0.08 ; \mathrm{RPE} 35 \% \mathrm{VO}_{2}$ peak: No diabetes, 11.1 $\pm 0.5, \mathrm{~T} 2 \mathrm{D}, 12.1 \pm 0.5, P=0.21$, presented as mean $\pm \mathrm{SD}$. T2D was associated with greater RPE at low intensity work rates, but not significantly so, despite worse $\mathrm{VO}_{2}$ peak levels in $\mathrm{T} 2 \mathrm{D}$ than controls. Although published $\mathrm{VO}_{2}$ peak differences exist in older and younger women with $\mathrm{T} 2 \mathrm{D}$ vs. their nondiabetic counterparts, we found less accentuated RPE differences by T2D status in older women than in our prior study of younger women. It appears that the effects of aging on RPE may exceed the effects of T2D-related exercise impairments in older adults.

Supported by: NIH/NCATS; Colorado CTSI KL2TR000156 (to A.G.H.)

2487-P0

Effects of Lifestyle Intervention for Veterans (LIVe) Program on Cardiometabolic Parameters in African American Veterans With Type 2 Diabetes Mellitus

SHIKHA KHOSLA, HAWAA ALMANSOURI, LAUREN KORSHAK, HELEN SHERIFF, MONICAAIKEN, KATHERINE HIMMERICK, IRUENAMATA-ELENGWE, RAYA KHEIRBEK, ERIC NYLEN, PETER KOKKINOS, Washington, DC

We developed a Lifestyle Intervention for Veterans (LIVe) Program to supplement medication therapy for diabetics. We assessed metabolic \& cardiovascular changes after a 12-week (wk) supervised exercise \& dietary intervention in African-American (AA) veterans.

All participants (age $=60 \pm 8.5 ; n=95$ ) had a peak exercise stress test, blood chemistries, resting and exercise blood pressure at baseline and after 12 weeks of exercise training. Participants were enrolled in supervised on-site or home-based exercise arms. On-site arm consisted of biweekly sessions of aerobic and resistance exercise prescribed by exercise physiologist with exercise intensity at $50-80 \%$ of heart rate reserve. Participants were asked to also brisk walk daily to accumulate $\geq 150$ minutes/wk of exercise. Homebased arm participants met monthly with exercise physiologist \& were asked to accumulate 150 minutes/wk of moderate intensity exercise.

On-site arm had significant improvements in exercise capacity, weight, waist circumference, cardiovascular and metabolic parameters (Table). No significant differences were observed in the home-based arm.

\begin{tabular}{lccc}
\hline VARIABLES & BASELINE & POST-EXERCISE & P-values \\
\hline Wt (lbs) & $230 \pm 43$ & $226 \pm 42$ & $<0.001$ \\
\hline Waist (inches) & $45.0 \pm 5$ & $44.0 \pm 5$ & 0.04 \\
\hline Rest SBP $(\mathrm{mm} \mathrm{Hg})$ & $126 \pm 17$ & $120 \pm 14$ & 0.001 \\
\hline Rest DBP $(\mathrm{mm} \mathrm{Hg})$ & $72 \pm 10$ & $68 \pm 9$ & 0.007 \\
\hline HR at $3 \mathrm{~min} .(\mathrm{bpm})$ & $110 \pm 14$ & $107 \pm 14$ & 0.02 \\
\hline SBP at 3 min. $(\mathrm{mm} \mathrm{Hg})$ & $158 \pm 20$ & $147 \pm 20$ & $<0.001$ \\
\hline DBP at $3 \mathrm{~min} .(\mathrm{mm} \mathrm{Hg})$ & $79 \pm 17$ & $73 \pm 10$ & 0.02 \\
\hline Peak HR (bpm) & $141 \pm 20$ & $143 \pm 20$ & 0.8 \\
\hline Peak SBP $(\mathrm{mm} \mathrm{Hg})$ & $191 \pm 24$ & $185 \pm 25$ & 0.03 \\
\hline Peak DBP $(\mathrm{mm} \mathrm{Hg})$ & $85 \pm 13$ & $80 \pm 12$ & 0.005 \\
\hline Peak METs & $8.7 \pm 1.8$ & $9.9 \pm 2.1$ & $<0.001$ \\
\hline Fasting Glucose $(\mathrm{mg} / \mathrm{dl})$ & $134 \pm 35$ & $115 \pm 30$ & 0.004 \\
\hline Hgb A1C & $7.6 \pm 1.5$ & $7.3 \pm 1.2$ & 0.03 \\
\hline Cholesterol $(\mathrm{mg} / \mathrm{dl})$ & $151 \pm 33$ & $146 \pm 30$ & 0.04 \\
\hline LDL-C $(\mathrm{mg} / \mathrm{dl})$ & $81 \pm 20$ & $81 \pm 25$ & 0.5 \\
\hline HDL-C $(\mathrm{mg} / \mathrm{dl})$ & $40.5 \pm 15.9$ & $40 \pm 15.6$ & 0.4 \\
\hline
\end{tabular}

Exercise Prevents Dysglycemia in Young Age

2488-PO

JOHN DOUPIS, ALEXANDER KOKKINOS, CHRIS DOUPIS, VASILEIOS ANDRIANESIS, ARTEMIS TRIKOLA, DESPOINA PERREA, NICHOLAS KATSILAMBROS, NICHOLAS TENTOLOURIS, Salamis, Greece, Athens, Greece

Impaired fasting glucose (IFG) is associated with the development of type 2 diabetes mellitus. In the present cross-sectional study we examined factors associated with impaired fasting glucose (IFG) in a cohort of 892 young subjects (mean age $22.2 \pm 2.9$ years; 60 females and 832 males). We excluded participants with known diabetes mellitus and those having fasting blood glucose $\geq 126 \mathrm{mg} / \mathrm{dl}$. Body weight and height was measured in light clothing and BMI was calculated. A validated structured questionnaire was applied to collect data for food consumption, physical exercise, history of type 2 diabetes mellitus, and smoking habits. Adherence to the Mediterranean diet was calculated using the Mediterranean diet score. A total of $145(16.3 \%)$ participants were found with IFG. Multivariate logistic regression analysis after adjustment for age, gender, BMI and family history of type 2 diabetes, 


\section{NUTRITION-CLINICAL}

demonstrated that the odds [odds ratio ( $95 \%$ confidence intervals)] of IFG reduced with frequent exercise $(0.92(0.74-1.00, p=0.05)$ but it was not associated with consumption of fruits and vegetables, meat, fish, cheese, coffee, beverages, saturated fat, fast-food, smoking or adoption of Mediterranean diet. Our data suggest that exercise is the only factor that protects from development of dysglycemia in young age.

2489-P0

Community-Based Dance Program: Effects on Diabetes and Obesity Prevention and Management

THERESA GARNERO, KATIE SAVIN, San Francisco, CA

This study evaluated the non-profit Dance Out Diabetes' Dance Off the Weight Program for its potential to promote exercise, improve healthcare outcomes and access to care, and reduce isolation among people with or at-risk for diabetes by including their immediate circle of support. The program entailed attending a fun, monthly cultural odyssey of dance classes taught by experts with built in health screenings and consultations with certified diabetes educations and registered dietitians, all at no cost to participants. Weight management was the educational focus for the year. Referrals to local primary care physicians, endocrinologists and social worker consultation were also available. The program was primarily volunteer run and hosted by a local community center. Health metrics captured included BMI, waist circumference, blood pressure, A1C, pre- and post-dance blood glucose levels, selfreported exercise intensity/duration and eating habits.

Out of 301 total participants who were primarily women belonging to diverse ethnic backgrounds and included all age groups, 203 opted to have their metrics recorded. From month to month, the retention rate was $25 \%$ with a total of 29 participants who did complete re-resting of all metrics. Most significantly, analysis of this initial data showed a mean A1C level drop of .49\% and a mean BMI decrease of 1.28. A third of participants were support people, such as friends and family, who indicated that they do not have diabetes.

Despite limitations in sample size and the potential for a social desirability bias amongst those who opted out of metrics recording, the improvements in A1c and BMI values are significant and warrant further investigation. The program provided a monthly intervention, yet potentially modified behaviors to promote health between sessions. Ongoing data will continue to be evaluated to determine if a monthly, cost-effective, community-based dance program is effective in preventing and managing diabetes and obesity.

Supported by: Aetna Foundation

\section{NUTRITION-CLINICAL}

\section{WITHDRAWN}

2491-P0

Reversal of Type 2 Diabetes to Normal by Self-Care: Motivation by Knowledge

SARAH STEVEN, EE LIN LIM, ROY TAYLOR, Newcastle upon Tyne, United Kingdom

The Counterpoint Study demonstrated regain of normal beta cell function and normal blood glucose control in type 2 diabetes following hypocaloric diet to achieve $15 \%$ weight loss. Widespread media coverage of precipitated many enquiries, and information was posted online. The aim of the present study was to collate the spontaneous feedback of individual experiences of sustained calorie restriction achieved outside a research setting by selfselected motivated individuals attempting to reverse their type 2 diabetes. Emails, letters and telephone communications received were evaluated $(n=77$ : M66, F11). Median diabetes duration was 5.5 years (3 months -28.0 years). Reversal of diabetes was defined as achieving fasting capillary blood glucose $<6.1 \mathrm{mmol} / \mathrm{I}$ and/or, if available, HbA1c of $6.1 \%$ or less off treatment.

Weight fell $14.8 \mathrm{~kg}(96.7 \pm 17.5 \mathrm{~kg}$ to $81.9 \pm 14.8 \mathrm{~kg} ; \mathrm{p}<0.001)$. Fasting glucose levels fell from $8.3(5.9-33.0) \mathrm{mmol} / \mathrm{l}$ to $5.5(4.0-10.0) \mathrm{mmol} / \mathrm{l}$ after the weight loss period $(p<0.001)$. Diabetes reversal occurred in $61 \%$ of the population. Reversal of diabetes was observed in $80 \%$; $63 \%$; and $53 \%$ of those with $>20 \mathrm{~kg} ; 10-20 \mathrm{~kg}$; and $<10 \mathrm{~kg}$ weight loss respectively. There was a significant correlation between degree of weight loss and fasting glucose levels (Rs-0.38 $p=0.006)$. Reversal rates according to diabetes duration were: $73 \%$ in shor $(<4 y), 56 \%$ in medium (4-8y) and $43 \%$ in long ( $>8 y)$ duration diabetes. Ora hypoglycemic agents and insulin were stopped when hypocaloric dieting began. Profound relief at being able to take personal action about a disease labeled as incurable was reported by all. Longer term follow up data were available on nine individuals, and these demonstrated sustained reversal of type 2 diabetes over a median of 5 months despite modest weight gain $(+2.4 \mathrm{~kg})$.

These data demonstrate that weight loss achieved at home by healthmotivated individuals reverses type 2 diabetes commonly. Type 2 diabetes is a potentially reversible condition.

Supported by: Diabetes UK

2492-P0

Relationships Between Changes in Composition of Major Fatty Acids and Changes in Fat Distribution, and Insulin Resistance in Japanese

TOSHIHIDE KAWAI, YUKO OGUMA, FUMINORI KATSUKAWA, KOICHIRO AZUMA KUMIKO TANAKA, YOSHIFUMI SAISHO, SHU MEGURO, HIDEO MATSUMOTO, HIROSHI ITOH, Tokyo, Japan

We have previously reported that serum linoleic acid level was inversely correlated with accumulation of visceral fat $(n=83, r=-0.455, p<0.0001)$ in relation to a reduction of insulin resistance in a cross-sectional study. This study was a longitudinal evaluation of the relationships between changes in the composition of free fatty acids (FFA) and changes in metabolic parameters, including body fat distribution. The study subjects were 12 Japanese $(6$ male and 6 female; mean $\pm S D$ for age $47.7 \pm 11.2$ years) who underwent $75 \mathrm{~g}$-OGTT. $\mathrm{BMI}$, blood chemistry, 24 fractions of fasting FFA, and visceral and subcutaneous fat areas as determined by CT scanning were measured at the beginning and after $1.96 \pm 1.58$ years. All parameters did not change significantly: $97.8 \pm 10.9$ to $101.3 \pm 17.2 \mathrm{mg} / \mathrm{dl}(\mathrm{p}=0.359)$ in FPG, $5.5 \pm 0.3$ to $5.9 \pm 1.1 \%(\mathrm{p}=0.122)$ in HbA1c, $7.25 \pm 5.73$ to $6.91 \pm 5.23(p=0.388)$ in ISI (comp), $99.8 \pm 47.3$ to $108.6 \pm 53.4 \mathrm{~cm} 2(\mathrm{p}=0.203)$ in visceral fat area, $1.16 \pm 0.24$ to $1.09 \pm 0.27(\mathrm{p}=0.503)$ in $\mathrm{L} / \mathrm{S}, 33.0 \pm 4.3$ to $30.0 \pm 6.2 \%(\mathrm{p}=0.182)$ in linoleic acid, $22.5 \pm 1.5$ to $22.8 \pm 3.2 \%$ $(p=0.938)$ in palmitic acid, $19.7 \pm 2.1$ to $19.0 \pm 3.7 \%(p=0.480)$ in oleic acid. However, $\Delta$ linoleic acid were inversely correlated with $\Delta$ visceral fat, positively correlated with $\Delta \mathrm{L} / \mathrm{S}$ and with $\Delta \mathrm{ISI}$ (comp). In conclusion, these data suggest that the changes in linoleic acid level were inversely correlated with the changes in visceral fat in relation to insulin resistance in Japanese.

\begin{tabular}{|c|c|c|c|c|}
\hline & \multicolumn{2}{|c|}{$\Delta$ visceral fat $\Delta$ subcutaneous fat } & \multirow{2}{*}{$\frac{\Delta \mathrm{L} / \mathrm{S}}{0.621}$} & \multirow{2}{*}{$\frac{\Delta \mathrm{ISI} \text { (comp) }}{0.611}$} \\
\hline$\Delta$ Linoleic acid (\%) & -0.693 & -0.488 & & \\
\hline & 0.016 & 0.132 & 0.040 & 0.033 \\
\hline \multirow[t]{2}{*}{$\Delta$ Palmitic acid (\%) } & 0.538 & 0.475 & -0.66 & -0.385 \\
\hline & 0.089 & 0.144 & 0.024 & 0.223 \\
\hline \multirow[t]{2}{*}{$\Delta$ Oleic acid (\%) } & 0.313 & -0.184 & 0.059 & 0.176 \\
\hline & 0.359 & 0.598 & 0.868 & 0.593 \\
\hline
\end{tabular}

Supported by: MEXT (Japan) 
How Many Calories Do We Consume During Christmas Holidays? MALGORZATA U. NAPIERALA, MARTAE. BRYŚKIEWICZ, KRZYSZTOF SAFRANOW, LILIANNA MAJKOWSKA, Police, Poland, Szczecin, Poland

Due to the tradition of family feasting, many people gain weight during the holidays. There have been only a few isolated reports on this problem. The aim of this study was to evaluate the increase of body mass, as well as the calorie intake and composition of the diet of healthy subjects during the Christmas holidays.

The study group included 30 subjects, all recruited from the employees of the University Hospital (13 members of medical staff and 17 administrative workers). For all of them, we scheduled measurement of body mass and composition performed using the Tanita Bc-418 analyzer before Christmas 2010 and immediately after the holidays. The participants were asked to note down the food and drinks consumed during the holiday season (in Poland 24-26 Dec). Data from the food diaries were evaluated by a dietician and entered into a computer program for analysis of diet composition and statistical analysis.

The final analysis was carried out for 23 subjects (7 did not report for the follow-up visit after the holidays), 22 subjects submitted dietary records. The observed mean body mass increase was $0,5 \pm 06 \mathrm{~kg}(p<0,0001)$, and the analysis of body composition demonstrated it was due to an increase in fat mass. A weight increase of $0,5 \mathrm{~kg}$ during 3 days of holidays required the consumption of 3500 extra calories, that is approximately $1160 \mathrm{kcal}$ above norm daily. Analysis of the food diaries showed that the mean energy intake equaled $2300 \pm 640 \mathrm{kcal}$ a day and exceeded the subjects' daily basal metabolic rate (BMR) by approximately $930 \mathrm{kcal}$. The source of the excess energetic value were mainly consumed fats.

1. The increase of body mass in the hospital employees during Christmas holidays was lower than expected. 2. The energetic value and composition of the holiday diet reported in the food diaries were quite accurate as to the amount of calories consumed. 3 . The results could have been influenced by the fact that the study was performed in hospital employees and the work of some of them involves adjusting appropriate diet for patients.

\section{PSYCHOSOCIAL, BEHAVIORAL MEDICINE}

2494-PO

WITHDRAWN
2495-P0

"Es como una bomba de tiempo (It's like a time-bomb)" A Qualitative Analysis of Perceptions and Knowledge among First Degree Relatives of Latino Diabetic Patients

ERIDA VASQUEZ, MARIA MILAN, BALAVENKATESH KANNA, CARLA BOUTINFOSTER, Bronx, NY, New York, NY

The South Bronx, a largely Latino community has become an epicenter of the diabetes epidemic in New York City. In this community, non-diabetic first degree relatives of patients with diabetes are prime targets for intervention to prevent diabetes.Participants were recruited from 3 settings in the South Bronx (a community-based organization, a faith-based organization, and a taxi cab station). The Common Sense Model was used to develop focus groups items. This model provides a framework for exploring illness representations along 5 domains identity, cause, consequences, timeline, and perceptions of curability.

A total of 115 potential participants were screened and 23 had a first degree relative with diabetes but they themselves did not have a diagnosis and thus, were eligible. Of these, 20 were Dominicans, 2 Puerto Ricans and 1 Salvadorian. The mean age was 46.39 years, $35 \%$ were women, $61 \%$ were married and $26 \%$ had less than a high school education. Qualitative analyses resulted in 547 codes which were grouped into 52 concepts from which 9 categories and 4 overarching themes emerged. The dominant themes were 1) Diabetes was a devastating and destructive condition with physical and emotional consequences 2) Diabetes could be prevented through healthy eating and could 'cured' 3) FamilylGenetics and culture, played a major role in etiology and prevention of diabetes 4) Being Latino and having a first degree relative with diabetes made getting diabetes inevitable- like a time bomb exploding, it is destined to happen.In this study, first degree relatives of patients with diabetes were knowledgeable of the risks of diabetes. Some participants felt that being Latino and having a first degree relative with diabetes made one destined to have diabetes. Addressing this misperception through culturally tailored interventions may help to stem the diabetes epidemic in Latino communities and reduce the risk among first degree relatives.

2496-P0

\section{WITHDRAWN}


2497-PO Improvement of Glycemic Control after Scheduled Follow-Up in Type 1 Diabetic Patients

JINHUA YAN, XIAOHUANXING, DAIZHIYANG, WENXU, JIN LI, BIN YAO, GUOCHAO

ZHANG, JIANPING WENG, Guangzhou, China

Type 1 diabetic (T1D) patient is difficult to achieved good glycemic control. Our previous first-phase study also showed poor glycemic control in Guangdong T1D patients. Therefore, in the second-phase of our study scheduled follow-ups were initiated. The aim of the study was to investigate the effect of the scheduled plan and analyze the related factors.

From June 2011 to December 2012, 144 T1D patients [age 29.0 yrs (22.0$38.0)$, duration of disease $4.5 \mathrm{yrs}(2.8-9.0)$, body mass index $19.8 \mathrm{~kg} / \mathrm{m}^{2}(18.9$ 22.0), male/female 64/80] [median (interquartile range)] were enrolled and followed up every 3 month in our hospital. Diabetic education, insulin dose titration and free $\mathrm{HbA1c}$ test were provided in each visit.

At baseline, HbA1c was 8.2\% (6.7-9.8) and 43 patients (28.5\%) achieved the age-specific targets. The number of patients completing the 3-, 6-, 9- and 12 -month visit was $97,50,44$ and 36 , respectively. $50.5 \%, 80 \%, 77 \%, 63.9 \%$ of patients reached the age-specific glycaemic goals at the 3-, 6-, 9- and 12 -month visit, respectively. There was a significant improvement in glycemic control with $\mathrm{HbA1c}$ falling from 8.2 (6.7-9.8) to 7.2\% (6.4-8.3) at 3-month, $7.2 \%$ (6.6-7.9) at 6-month, 7.0\% (6.4-7.7) at 9-month and 7.0\% (6.5-8.0) at 12-month $(\mathrm{P}<0.05$ compared with baseline). Binary logic regression analysis showed that patients with more frequent self-monitoring of blood glucose (SMBG) $[95 \% \mathrm{Cl}(1.076,2.039)]$ and those with diabetic duration (1-10 years) [95\% Cl $(1.002,11.028)]$ had better glycemic control.

In summary, our results indicated that scheduled follow-up has a favorable effect on glycemic control in Guangdong T1D patients. Higher frequency of SMBG is recommended for glycemic improvement.

Supported by: Science and Technology Planning Project of Guangdong (2010B31500008)

2498-P0

Lifetime Racial Discrimination Predicts Behavioral and Cardiovascular Reactivity to Acute Stress in Women With Type 2

JULIE A. WAGNER, HOWARD TENNEN, RICHARD FEINN, ANGELA BERMUDEZMILLAN, Farmington, CT, Hamden, CT

Exposure to racial discrimination has been linked to adverse health outcomes. In a multi-racial sample of women with type 2 diabetes (T2DM), this study investigated racial discrimination and heart rate (HR) and eating following an acute laboratory stressor. Lifetime exposure to racial discrimination was measured with the Schedule of Racist Events (Landrine 1996). HR was measured with a Welch Allyn vital signs monitor. Same-day eating behaviors were per self-report. 77 women (51\% African-American, 49\% White) with T2DM fasted for 8 hours, then ate a standardized breakfast and completed a resting baseline, followed by 6-min public speaking stressor. HR was measured at baseline, during stress, and recovery. Participants left the laboratory at noon. That evening, participants completed self-reports of same-day eating. Measures were repeated on a counterbalanced control day. Women were $M=56 \pm 12$ years old, with T2DM for $9 \pm 8$ years, on oral agents $55 \%$, with adequate glycemic control $(A 1 c=6.8 \pm 2)$. On control day, there was no effect for discrimination $(\mathrm{p}=.85)$ or a discrimination $\mathrm{x}$ time interaction $(\mathrm{p}=.93)$ on HR. On stressor day, there was a discrimination $x$ time interaction $\left({ }^{*} p=.019\right)$. Higher discrimination predicted slower HR recovery from the public speaking challenge, even after accounting for baseline HR. Findings held after controlling for race, duration of diabetes, and trait hostility. On control day, there was no effect for discrimination on eating $(\mathrm{p}=.20)$. On stressor day, higher discrimination predicted more out of control eating, even after accounting for out of control eating on control day $\left({ }^{*} \mathrm{p}=.04\right)$. Findings held after controlling for race and hostility $\left({ }^{*} p=.03\right)$. Exposure to racial discrimination may impair cardiovascular functioning and increase appetitive behaviors in response to acute stress. If findings are replicated in a larger sample, behavioral interventions for women with T2DM should address racial discrimination.

Supported by: NIDDK

2499-P0

Oral Health Related Quality of Life in Diabetic Subjects in Mexico City

SOCORRO AIDA BORGES-YAÑEZ, ROBERTO CARLOS CASTREJÓN-PÉREZ, GUADALUPE FABIAN-SAN MIGUEL, Mexico City, Mexico

Oral health problems in diabetic persons have an impact on quality of life $(0 \mathrm{~L})$, loss of teeth and periodontitis are common problems in diabetics when no self and professional oral care is provided. Objective: to estimate the impact of oral health conditions on $\mathrm{QoL}$ in a group of diabetic persons. Meth- ods: Crossectional study. 247 diabetics persons with $\geq 10$ years of progression participants in the " $19^{\text {th }}$ annual walk of the diabetic patient" organized by the National Institute of Respiratory Diseases in Mexico City. Variables: OHROOL (Oral Health Impact Profile OHIP-14 validated in México), self-perception of oral health, age, time from diagnosis, number of teeth, xerostomia, selfperception of need for dental treatment (NDT), knowledge of special dental care, knowledge of the effect of diabetes on oral health, loose teeth, acute dental pain, diagnosis of periodontitis halitosis, root remains (RR), smoking Analysis: Univariate generalized linear model (GLM) to identify the variables associated with $\mathrm{OHROOL}$ controlling by sex, smoking and time of progression. Results: Mean age was 63.8ะ9.0, (range 34-86), 20.6\% were males. No differences were observed in the median OHIP-14 by gender and age group. In the univariate analysis, the persons who perceived their oral health as bad had a significant higher OHIP-14 median (22), those with diagnosis of periodontitis (median=20), with acute teeth pain (median=16), loose teeth (median=17), halitosis (median=13), partially dentate (median=13) and with RR (median=17) had worse OHROoL. The GLM showed that for every tooth present the OHIP-14 score decreases 0.35 units, a good self-perception of oral health decreases in 10.9 units, to have no loose teeth, does not have halitosis or does not consider to NDT the OHIP-14 scores decreases 5.7, 4.8 and 5.0 units respectively (adjusted $\mathrm{R} 2=0.36$ ). Conclusion. Oral health problems in diabetic subjects have a negative impact on quality of life.

2500-P0

\section{WITHDRAWN}

\section{WITHDRAWN}


2502-P0

Eating Disorder Prodromal Factors in People With Type 1 Diabetes MARGARET A. POWERS, SARA RICHTER, DIANN ACKARD, CHERYL E. CRAFT, REBECCA PASSI, Minneapolis, MN

Eating disorders have the highest rates of mortality and both medical and psychiatric comorbidity of all of the psychiatric disorders. This study examined prodromal factors related to eating disorders among patients with T1D. Participants were 278 patients with T1D, 58\% female; $67 \%<18 \mathrm{yrs}$; mean BMI 23.6 (SD \pm 4.6 ): mean yrs of diabetes diagnosis 10.3 yrs (range 1-50 yrs). We found that $23 \%$ of those $<18 \mathrm{yrs}$ and $38 \% \geq 18 \mathrm{yrs}$ were dissatisfied with their looks. After controlling for BMI, those dissatisfied with how they looked had significantly higher diabetes distress, less satisfaction with life, lower selfesteem, less self-efficacy, more depressive symptoms, higher eating disorder symptoms (dietary restriction, and concerns about eating, shape, weight). There was no difference in the A1c of those dissatisfied or satisfied with their looks. The dissatisfied individuals are struggling with a greater constellation of psychological concerns that are known to be associated with the later development of an eating disorder. These findings suggest that about $23 \%$ of youth and $38 \%$ of adults with T1D may be experiencing prodromal factors to the onset of an eating disorder. Diabetes clinicians should be prepared to address the convergence of these numerous emotional factors and have a plan for prevention and early intervention.

\begin{tabular}{|c|c|c|c|c|c|}
\hline & \multicolumn{5}{|c|}{ Age Category } \\
\hline & \multicolumn{3}{|c|}{$<18$ years } & \multicolumn{2}{|c|}{$>=18$ years } \\
\hline & \multicolumn{5}{|c|}{ Satisfaction with Looks } \\
\hline Variable & Dissatisfied & Satisfied & $p$ & Dissatisfied & Satisfied \\
\hline Current Age (yrs) & 15.1 & 14.6 & & 33.1 & 25.1 \\
\hline Age at DM Diagnosis (yrs) & 7.2 & 7.3 & & 14.1 & 10.5 \\
\hline Yrs with DM & 7.9 & 7.3 & & 19.0 & 14.5 \\
\hline A1c & 8.78 & 8.68 & & 8.28 & 7.82 \\
\hline BMl & 24.8 & 21.6 & * & 30.0 & 23.8 \\
\hline BSRS - Current Body Shape & 4.6 & 3.7 & * & 5.3 & 4.0 \\
\hline BSRS - Ideal Body Shape & 3.1 & 3.4 & * & 3.6 & 3.6 \\
\hline DDS2 (Diabetes Distress) & 3.5 & 2.4 & * & 3.6 & 2.5 \\
\hline Satisfaction with Life Scale & 18.3 & 27.2 & * & 17.4 & 26.4 \\
\hline Rosenberg Self-Esteem Scale & 14.3 & 23.4 & * & 14.8 & 22.3 \\
\hline General Self-Efficacy Scale & 27.6 & 32.0 & * & 27.8 & 33.5 \\
\hline CES-D Score (Depression) & 20.2 & 6.6 & * & 20.3 & 6.8 \\
\hline EDE-Q Global Score (Eating Disorder) & 2.8 & 0.5 & * & 2.7 & 0.8 \\
\hline MPS Global Score (Perfectionism) & 109.4 & 92.5 & * & 108.5 & 99.3 \\
\hline
\end{tabular}

Means are reported in this table. ${ }^{*}=p$-value $<0.001$

Supported by: Park Nicollet Foundation

2503-P0

Qualitative Insights into Psychosocial Needs and Strategies of People With Diabetes and Family Members in DAWN2 Study HEATHER STUCKEY, KATHARINA KOVACS BURNS, HITOSHI ISHII, SANJAY KALRA, CHRISTINE MULLAN-JENSEN, ANTONIO NICOLUCCI, GÉRARD REACH, SØREN SKOVLUND, MICHAEL VALLIS, JOHAN WENS, INGRID WILLAING, MARK PEYROT, ON BEHALF OF THE DAWN2 STUDY GROUP, Hershey, PA, Edmonton, AB, Canada, Tenri, Japan, Karnal, India, Bagsvaerd, Denmark, Santa Maria Imbaro, Italy, Paris, France, Halifax, NS, Canada, Antwerp, Belgium, Gentofte, Denmark, Baltimore, MD

To identify perceptions of the positive and negative aspects of living with diabetes, the second Diabetes Attitudes, Wishes and Needs (DAWN2) study surveyed 8586 adults with diabetes (1358 with T1 and 7228 T2), and 2057 family members (FMs) of people with diabetes (PWD) in 17 countries across
4 continents. Qualitative data, drawn from responses of FMs and PWD to open-ended survey questions, were thematically analyzed.

Worry about hypoglycemia was a common concern to PWD and FMs. PWD worried about needing to be treated for hypoglycemia by others, while FMs were distressed by severe episodes requiring treatment by FMs or hospital. FMs were frightened by "my wife speaking nonsense in the middle of the night" or because "my mother did not know me."

PWD described the emotional impact of diagnosis as depressing, "the worst," and shocking. Anxiety and the difficulty of mentally living with diabetes as a "constant fight" were common to PWD. One PWD said, "Living with diabetes is like living with a pet rattlesnake - you can feed and care for the snake, but it may still bite you." The support of others was the most often reported success for PWD, with some family relationships strengthened "more than before," or becoming closer, as in "the whole family is diabetic." Tensions also existed for the FMs to "deal with what needs to be done day by day." Some FMs felt helpless as the PWD rejected advice.

Many PWD found success by persisting in treatment and "fighting the disease with a good spirit," integrating diabetes into everyday life, or expressing happiness to be alive. For FMs, living with PWD was said to be inspirational, teaching FMs to "look after my own health," so "both eat a healthier diet." Wishes for improvement include PWD taking better control through losing weight and improved eating or exercise habits. Ultimately, both PWD and FMs wished for a cure.

The personal narratives of PWD and FMs give insight into their psychosocial needs and coping strategies, and can inform global action programs.

$\Delta$ 2504-P0

Self-Administered Diabetes Self-Management Profile (SA-DSMP): Reliability, Validity, and Utility

MICHAEL A. HARRIS, DANNY C. DUKE, JENNIFER K. RAYMOND, JONAH B. HARRIS, LISA SHIMOMAEDA, KEVIN SHIMOMAEDA, Portland, OR

The Diabetes Self Management Profile (DSMP) is a widely used measure of diabetes self-management of youth with type 1 diabetes. Spanish, shortened, and self-report versions of the DSMP have been developed. However, to date, the validity of the DSMP as a self-administered interview has not been examined.

The DSMP was modified into a self-administered iPad-based format (SelfAdministered DSMP; SA-DSMP) and administered to 53 youth with type 1 diabetes and their caregivers. Changes included only minor language alterations to improve readability. Mothers comprised $77 \%$ of the caregiver respondents. Mean youth age was 15.3 years $(S D=1.7)$ and $49 \%$ were female. Mean duration of diabetes was 6.6 years $(S D=4.3$ ) and mean $\mathrm{HbA1c}$ was $8.7 \%$ $(S D=1.7 \%)$. Approximately $84 \%$ of youth were Caucasian.

The SA-DSMP demonstrated adequate internal consistency (youth report $r=.76$, parent report $r=.85)$ and parent-youth agreement $(r=.71)$. The mean total SA-DSMP scores of youths and parents was 58 . For example, previous mean total DSMP scores for youth were 54.3 and parent reports were 60.3 when administered by a trained interviewer.

SA-DSMP total scores correlated significantly with $\mathrm{HbA1c}$ (parent $r=-0.59$, youth $r=-.44$ ) as did subscale scores (Diet [parent $r=-.62$, youth $r=-.35$ ], Insulin Administration [parent $r=.-50$, youth $r=-.54$ ], Hypoglycemia Management [parent $r=-.39$, youth $r=-.24$ ], and BG testing [parent $r=-.36$ ]). In a prior study, the correlation between the DSMP and HbA1c was $r=-.28, p<.01$. These findings indicate the DSMP can be reliably used in its current format, but self-administered. Findings also indicate that in a typical clinical population of youth with T1DM the SA-DSMP yields similar means to trained interviewer administration. Finally, the relationship between the SA-DSMP and $\mathrm{HbA1C}$ remained strong. This study of the SA-DSMP offers preliminary evidence in support of self administration. Advantages include reduced costs and time resulting by reducing need for trained interviewers.

2505-PO

Cognitive Coping and Diabetes Distress in Treated Adults With Type 2 Diabetes

MOLLY L. TANENBAUM, JEFFREY S. GONZALEZ, Bronx, NY

Diabetes distress is common and has been linked to worse glycemic control, poorer self-management and reduced quality of life for individuals with type 2 diabetes. A better understanding of the psychological processes related to the experience of diabetes distress could aid in identifying points of therapeutic intervention. Therefore, we evaluated patient reports of typical cognitive strategies for coping with stressful life events (Cognitive Emotion Regulation Questionnaire); the tendency to respond toward oneself with mindfulness, kindness, and understanding during difficult times (Self-Compassion Scale); and diabetes distress (Diabetes Distress Scale) in a cross- 


\section{PSYCHOSOCIAL, BEHAVIORAL MEDICINE}

sectional study of 121 adults receiving treatment for type 2 diabetes (M age $=56.2, S D=9.67 ; 63.6 \%$ female; $61.2 \%$ Black; $25.6 \%$ Latino). A hierarchical multiple regression controlling for age, income level, and diabetes complications found that greater self-compassion ( $\beta=-.21, p=.03$ ), and less other-blame $(\beta=.24, p=.02)$ were associated with lower levels of diabetes distress. Although a composite of rumination, catastrophizing and self-blame was initially positively associated with diabetes distress $(\beta=.34, p<.001)$, this relationship was reduced to non-significance by self-compassion. Other strategies such as positive refocusing, perspective-taking, and acceptance were not linked with diabetes distress. Results suggest independent links between patients' cognitive approaches to coping with stressful life events and the experience of diabetes-related distress. Pending longitudinal analyses will clarify the directionality of these relationships and could inform interventions aimed at reducing diabetes distress.

2506-PO

Barriers to Addressing Mental Health Problems-Diabetologists' Views on Routine Diabetes Consultations

CLEA B. JOHANSEN, RIKKE TORENHOLT, EVA E. HOMMEL, MINNA WITTRUP, INGRID WILLAING, Gentofte, Denmark

Diabetes is associated with an increased risk of diabetes distress, depression, anxiety and eating disorders. Apart from being important in their own right, mental health problems are associated with poor glycemic control and increased mortality. However, health professionals working with diabetes fail to identify two out of three patients with serious mental health problems.

We explored diabetologists' self-perceived barriers to addressing mental health issues in routine consultations with patients with diabetes.

We conducted qualitative semi-structured individual interviews (50-60 minutes) with 12 diabetologists working in specialist diabetes clinics in four different Danish hospitals. The interviews focused on the physicians' perception of the importance in diabetes consultations of patients' mental health status, on physician perceptions of barriers to addressing mental health status, and individual physician experiences with addressing mental health issues. All interviews were transcribed verbatim and analysed by meaning condensation and interpretation according to the method of Kvale and Georgi.

The perceived barriers were related to the health care system, to patient characteristics and to physician attitudes. Barriers relating to the health care system were for example limited time, demands of documentation and extensive filling out of electronic records. Patient-related barriers included patients appearing indifferent to or defeated by their diabetes. Barriers related to physician attitudes included lack of skills and the feeling of being 'overwhelmed' by the psychological burden of patients.

In conclusion diabetologists perceive considerable barriers to addressing mental health problems in diabetes consultations and do not experience support for systematically addressing mental health issues in diabetes through the health care system.

2507-PO

Assessing Psychopathology and Diabetes-Related Distress in a Pediatric Diabetes Population

ELIZABETHB. SIMMONS, SARAHBOSTICK, LAUREN LEGATO, MARIA D'ANIELLO, KATIE KASZYNSKI, LOUIS PHILIPSON, EMIL COCCARO, TINA DROSSOS, Chicago, IL

Previous literature has demonstrated a strong association between diabetes and increased risk for psychopathology, including anxiety, depression, and externalizing problems in pediatric populations. Additionally, research suggests that psychopathology is related to poorer metabolic control. Early detection of psychopathology among pediatric populations is essential to improve diabetes management. The Problem Areas in Diabetes Scale- 5 (PAID-5) is a brief assessment designed to detect diabetes-related distress. This study aims to examine the relationship between diabetes-related distress, as measured by the PAID-5, and psychopathology among a pediatric sample. Data was collected through semi-structured interviews conducted by the Health and Wellness team within the University of Chicago Kovler Diabetes Center. The current sample consists of 31 children ages 3 to $17(M=13.45, S D=3.63)$ with diabetes. Patients were ethnically diverse with 61\% Caucasian, 26\% African American, $10 \%$ other/biracial, and 3\% Hispanic. Bivariate correlations indicated that higher diabetes-related distress was associated with the endorsement of several psychological symptoms, including Generalized Anxiety Disorder $(r=.52, p<.01)$, Depression $(r=.36, p<.05)$, Attention Deficit Hyperactivity Disorder $(r=.34, p<.05)$, and Conduct Disorder $(r=.37$, $p<.05)$. This study highlights the relationship between diabetes-related dis- tress and psychopathology among children and adolescents with diabetes. Additionally, results suggest that the PAID-5 may be an effective, timely, and user-friendly screening tool to detect pediatric patients who warrant a more comprehensive psychological assessment and intervention plan to improve health outcomes.

2508-PO

Do Patients and Physicians Agree on Diabetes Management? A Study Conducted in Public Healthcare Centres in Brazil

ROBERTA C. FIGUEIREDO, FRANK J. SNOEK, SANDHI M. BARRETO, Belo Horizonte, Brazil, Amsterdam, Netherlands

The way physicians understand their patients' health beliefs and preferences is a fundamental feature of patient-centred care. Often physicians have limited awareness of the patients' perspectives, and this topic therefore constitutes an important field of research in diabetes care, both in developed and developing countries. We aimed to explore to what extent patients with diabetes agree with their physicians on diabetes management and whether the agreement varies according to patients' socio-demographic characteristics. A cross-sectional epidemiological study was conducted among patients with diabetes and their Family Health physicians in 108 healthcare centres in Belo Horizonte, Brazil. Data were gathered by face-to-face interviews Physicians were unaware of which of their patients would be interviewed. Responses were compared using descriptive statistics and Cohen's weighted kappa. 282 physician-patient pairs were included. The kappa coefficients were often low, the highest was found for presence of diabetic foot and the lowest for kidney disease. Physicians tended to overestimate patients' risk of diabetes complications and underestimate patients' adherence to all diabetes self-management activities as well as diabetes control. In addition, the agreement rate regarding adherence to diet, foot care and medicine prescriptions was significantly higher among male, younger and higher educated patients. Results indicate that physicians' recommendations are generally poorly apprehended by their patients, especially by the lower educated, compromising the goal of patient-centred care. Educational programmes need to incorporate strategies to improve the comprehension and effectiveness of physicianpatient communication, especially with the most socially vulnerable groups.

Supported by: FAPEMIG; CNPq

2509-P0

Empowering Young Women With Type 1 Diabetes: Evaluating a PeerLed Diabetes Support Group

KATIE SAVIN, New York, NY

This pilot study aims to assess the feasibility and acceptability of a peer-led support group for young women with type 1 diabetes - the first of its kind in current literature. We also explore the impact of support group attendance on group members' diabetes management and distress. The support group was run at no direct cost, using donated meeting space and volunteer time. Participants ( $n=14)$ were directed to the online survey via support group listserv emails and flyers. Qualitative outcomes were measured with open-ended questions and were analyzed using the thematic analysis approach to coding. Quantitative outcomes were measured using demographic information, selfreported A1C levels, and frequency of endocrinologist visits and support group meetings attended.

The overall impression of the group experience was that it filled a void in participants' diabetes care and that more frequent group meetings were desired. Qualitative responses revealed three major themes of group impact on participants: validation of diabetes-related struggles, reduction of isolation, and increases in perceived self-efficacy of diabetes management. Selfreported pre-support group $\mathrm{A} 1 \mathrm{C}$ ranged from $6.8 \%-13.1 \%$ with a mean of $8.7 \%$. The mean dropped to $8.0 \%$ with a range of $6.0 \%-10.0 \%$ after group attendance. Equal numbers of participants regularly went to an endocrinologist pre- and post- group attendance.

The findings of this novel study suggest psychosocial benefits for young women with type 1 diabetes resulting from attending a peer-led support group. Though self-report is a limitation, findings suggest that participation has the potential to lower A1C. This study is an important contribution to the literature because it assesses an under-researched yet potentially efficacious and cost-effective intervention. Results suggest that clinicians should include support and advocacy for starting peer-led groups. Larger scale and longitudinal research is needed to reinforce and further these initial findings. 
A Cross Cultural Comparison of Diabetes Related Distress in Patients With Type 2 Diabetes Mellitus: China and Germany

MINGZI LI, LINONG JI, NORBERT HERMANNS, Beijing, China, Bad Mergentheim, Germany

Diabetes related distress is a common experience of patients with diabetes, which includes high levels of negative affect and highly associated with depression and poor disease management. However, as Diabetes Distress Screening Scale (DDS) was developed in the western culture, does the culture difference affect its psychometric properties of the scale? Are there any difference in diabetes related distress across different cultures. Objectives of this study are to evaluate the structure validity of DDS in Chinese and German cultures, and compare its diabetes related distress across these two cultures. 360 and 100 T2DM patients were included from Diabetes center of Beijing and Bad Mergentheim accordingly. Results showed that 4 exactly the same factors were acquired in both Chinese and German patients with accumulated contribution rate of $62.6 \%$ and $68.9 \%$ accordingly. The AUC of DDS ROC curve in Chinese and German patients were 0.884(95\% Cl 0.831 0.937) and $0.836(95 \% C / 0.757 \sim 0.915)(P<0.001)$ accordingly. The DDS score of Chinese patients was significantly lower than that of German patients. Multiple linear stepwise regression analysis showed that depression state, nationality and gender entered the equation explaining $33.3 \%$ of DDS variation $(P<0.001)$.

Conclusion: DDS can serve as a tool for trans-cultural comparison. Depression state were the most important factor affecting the illness experience of patients in both cultures and the ability of predicting depression of DDS in both cultures was satisfactory.

2511-P0

Depression in Type 2 Diabetes Mellitus: Relationship With Gender, Habits, Glycemic Control and Diabetic Complications

SILVANA MILRAD, EVA LOPEZ GONZALEZ, ANGELA LUONGO, ALICIA GARCIA, MARIA LIDIA RUIZ MOROSINI, MARIA CRISTINA VARELA, SOLANGE HOUSSAY, FRADYC II GROUP, Buenos Aires, Argentina

Several publications suggested a relationship between type 2 diabetes (T2DM) and depression, being the underlying mechanisms still unclear. In 31 medical centers Specialized in Diabetes in Argentina, the frequency of depression symptoms in T2DM patients (pts) and its relationship with gender, habits, and glycemic control were evaluated. Depression symptoms were assessed with WHO- Five Wellbeing Index (WHO-5) considering abnormal 0 or 1 in any of the items surveyed or a total score less than $50 \%$. We followed 1491 pts, female $46.7 \%$, age $64.1 \pm 11.3$ years, diabetes duration $10.2 \pm 7.8$ years, BMI $31.4 \pm 6.0 \mathrm{~kg} / \mathrm{m} 2$, A1c $7.2 \pm 1.4 \%$, coronary disease $26.7 \%$ and neuropathy $30.2 \%$.

Mean WH0-5 test was $63.4 \pm 22.1 \%$; the Cronbach's alpha's was 0.81 confirming high internal consistency. Depression symptoms were present in $39.2 \%$ of pts, $16.4 \%$ had previous depression diagnosis.

Depression was associated with: female gender (OR:1.83, $\mathrm{p}<0.0001)$, neuropathy (OR: $1.35, p<0.009$ ), coronary disease $0 R: 1.38, p<0.03)$, unemployment (OR:1.38, p<0.002), smoking (0R:1.80,p<0.001). No association was found with: glycemic control A1c $>7 \%$ (0R:0.88, $p<0.26$ ), and insulin treatment (OR:1.19, $\mathrm{p}<0.91)$. Protective factors were: working (0R:0.72, $\mathrm{p}<0.002)$ and physical activity (OR: 0.49, p<0.0001).

Forward Stepwise Logistic regression was used to identify risk factors for depression: female gender (OR: 2.03; IC:1.62 - 2.55, p<0.0001), neuropathy (OR:1.29; IC:1.02-1.64, $\mathrm{p<0.03),} \mathrm{coronary} \mathrm{disease} \mathrm{(OR:1.34;} \mathrm{IC:1.01-1.81,} \mathrm{p}<0.04$ ), and smoking (OR: 1.92, Cl: 1.39-2.63, p<0.001). Physical activity persisted as a protective variable (OR: 0.63; Cl:0.51-0.78, p<0.0001).

Conclusions:Depression symptoms occurred frequently in this sample. Poor glycemic control was not associated with depression and physical activity was a protective factor. Female gender, neuropathy, coronary disease, and smoking were predictors for depression. Screening for depression may be necessary in T2DM. 
other demographic characteristics, and this may replicate on the quality of care provided by them. The objective of this present was to investigate whether the level of emotional distress of diabetic patients, rated by their physicians, is associated with patient race and neighborhood socioeconomic status. A cross-sectional study was conducted among diabetic patients and their Family Health physicians in 108 healthcare centres in Belo Horizonte, Brazil. Data were gathered by face-to-face interviews. Physicians were unaware of which of their patients would be interviewed. To identify the association between the response and explanatory variables, crude and adjusted odds rations were estimated by logistic regression. 282 physicianpatient pairs were included. After controlling for standard covariates, physicians rate black patients and those who live at neighbourhoods with high risk Health Vulnerability Index as having higher distressed than white patients (OR: 1.63; 95\% Cl: 1.01;3.98) who lived in a lower risk neighbourhood (OR: $1.50 ; 95 \% \mathrm{Cl}: 1.11 ; 2.43)$. The results support the hypothesis that physicians' perceptions of patients are influenced by patients' race and socio-economic status. In addition, if physicians' perceptions about the patients do affect quality of care as the existing literature suggests, our findings contribute, both, to explain the vicious circle of socioeconomic inequalities in diabetes related outcomes.

Supported by: FFAPEMIG; CNPq

\section{3-PO}

Lifestyle and Metabolic Assessment at a Group of Romanian Medical Students

SIMONA GEORGIANA G. POPA, BETTY BORNAGEL, GEORGIANA MUSTAFA, MARIA MOTA, Craiova, Romania

In the last years is registered an increased incidence of metabolic disease at ages increasingly smaller, principally due to lifestyle changes.

The aims of this study were the assessment of anthropometric and metabolic parameters and insulin resistance indices (Visceral Adiposity Index - VAl and Body Adiposity Index - BAl) at a group of Romanian medical students (group A) comparative with a group of subjects with higher degrees in nonmedical fields (group B) and also the evaluation of predictive factors for impaired glucose metabolism in analysed subjects.

This cross-sectional study included 171 subjects: 93 subjects in group A $(22.8 \pm 1.7$ years) and 78 subjects in group $B(29.3 \pm 4.8$ years $)$ in which were recorded lifestyle data, anthropometric and metabolic parameters, VAI and BAl.

Subjects of group B had an unhealthy lifestyle regarding diet and physical activity, compared with the group A. Sedentary life was correlated with abdominal and general obesity, increased LDL-C and VAl at all subjects.

The subjects from group $B$ had statistically significant higher values of waist, BMI, WHR, total cholesterol, LDL-C, triglycerides, fasting glucose, BAI and lower levels of $\mathrm{HDL}-\mathrm{C}$.

Prediabetes or diabetes were found at $52.6 \%$ of subjects from group $B$ and only at $12,9 \%$ of subjects from group $A$.

ROC curve analysis indicated the utility of VAl, age, lipid and anthropometric parameters in predicting the presence of impaired glucose metabolism. Of these parameters only WHR ( $p=0.01)$, total cholesterol $(p=0.02)$ and age $(p=0.005)$ were independent predictors for the presence of impaired glucose metabolism.

The access to information regarding healthy lifestyle of medical students could be an explanation for the existence of better anthropometric and metabolic parameters at medical students compared with the subjects from non-medical areas.

Although all subjects analyzed were young, the impaired glucose metabolism was present at $31 \%$ of them, and it was independently predicted by abdominal obesity, hypercholesterolemia and age.

\section{4-PO}

Physicians' Reports of Diabetes Patients' Distress Varies by Race and Socioeconomic Status

ROBERTA FIGUEIREDO, FRANK J. SNOEK, SANDHI M. BARRETO, Belo Horizonte,

Brazil, Amsterdam, Netherlands

It is well-established that emotional distress is common among people with diabetes and is associated with poorer treatment outcomes. However, emotional problems are often overlooked at physician-patient encounters, especially with less educated patients. Physician-patient interactions seem to be worse among poor, less educated and minority patients. There are evidences showing that differences in physicians' perceptions of their patients emotional distress may vary systematically by patient race, socio-economic status, or The Role of IIIness Beliefs and Social Networks in South Asian People With Diabetes: A Mixed Methods Study NEESHA R. PATEL, ANNE KENNEDY, ANNE ROGERS, CHRISTIAN BLICKEM, DAVID REEVES, CAROLYN CHEW-GRAHAM, Manchester, United Kingdom, Southampton, United Kingdom, Keele, United Kingdom

Diabetes affects 2.9 million people in the UK and is 6 times more common in South Asians (SA). Whilst beliefs have been reported to impede on selfmanagement behaviours, little is known about the role of Social Networks (SN), how much and what types of diabetes 'work' (the amount of support received from $\mathrm{SN}$ ) and the impact of $\mathrm{SN}$ in shaping beliefs about diabetes. The aim of this study was to gain knowledge about the link between illness beliefs and SN in SA adults with Type I or 2 diabetes. A Postal Health Survey with validated scales measured illness beliefs (Brief Illness Perception Questionnaire -BIPQ), health outcomes (health status, self-efficacy and self-care) and socio-demographics of ( $\mathrm{N}=67)$ SAs. Semi-structured, SN interviews included: identifying the people, groups and others (e.g. deities) in each person's network using concentric circles. Closed-questions on the amount of 'work' done by each SN member and a topic guide of open-ended questions explored social, cultural and religious beliefs related to diabetes ( $N=37)$. Data was collected during 2010 and 2011. Certain SN characteristics: size of the support network, number of supportive females, practical and emotional work was related to two BIPQ items. Emotional work remained a significant predictor of concern and emotional distress related to diabetes $(P<0.05)$. The $\mathrm{OS}$ identified 6 themes: fatalism, normalising diabetes, $S N$, alternative food therapies, and travel back home to the East and religion related to management practices; and complemented some of the findings of the cross-sectional study. Current policies for diabetes focus on supporting and empowering patients as individuals. This study provides a novel insight into whom, what and how much diabetes 'work' SN undertake, the role of cultural and religious beliefs; and questions whether guidelines and practitioners need to move beyond the notion of 'self' to include support for the SN, community and culturally-sensitive resources involved in diabetes management.

Supported by: NIHR; CLAHRC

\section{6-PO}

Diabetes Prevention: School Based Approaches in Oklahoma STEVEN STERNLOF, CYNTHIA MUHAMEDAGIC, AARON JENNINGS, Oklahoma City, OK

Obesity is among one of the most critical health issues facing our children and our society at the current time. While obesity shares a close relationship with the development of type 2 diabetes, prevention efforts can reduce the risk through changes in lifestyle behaviors. Prevention efforts to reduce the likelihood and risk of these problems have far reaching benefits above those of conventional treatments or waiting for problems to develop. Health education in the schools is one prevention model that serves as a powerful tool to change the habits of children and the community at large. Oklahoma has one of the highest rates of obesity in the nation as well as youth identified with in Oklahoma focuses on nutrition and physical activity that can reduce the development of type 2 diabetes. The Schools for Healthy Lifestyles Program

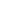




\section{CLINICAL THERAPEUTICS/NEW TECHNOLOGY—GLUCOSE MONITORING AND SENSING}

risk for obesity and diabetes. This program has been studied over the past seven years using a variety of statistical methods. Currently in more than 60 elementary schools across the state, the program has shown significant changes for participants in lifestyle behaviors, body mass index (BMI) and academic performance. A self report survey indicated that participants significantly changed their nutrition and physical activity patterns over the course of the program during the school year and also compared to participants and schools that did not participate in the program. Using the Fitnessgram as a measure of change pre to post, school participants in the overweight and obese classifications significantly reduced their BMI, and all participants increased cardiovascular levels of fitness as well as measures of strength. Lastly, participants in program schools significantly increased their academic performance over a span of five years compared to schools that did not have health promotion.

2517-P0

Is "Treatment-Resistant" Type 2 Diabetes Mellitus a Internal Disease? Psychiatric Approaches on Poor Adhearance as Origin of its Refractoriness

TETSUYA YAMAMOTO, AIZAN HIRAI, Chiba, Japan, Togane, Japan

In Japan, whereas patients requiring renal dialysis due to diabetic nephropathy represent only about $4 \%$ of total type 2 diabetes mellitus (T2DM) patients, their medical costs account for more than $40 \%$ of the total medical costs for T2DM, amounting to 13.7BUSD in 2008. Among around 1,000 T2DM patients we manage in our hospital, about $10 \%$ of them have kept poor glycemic control due to poor adherence despite that appropriate pharmacology, diet and exercise therapy have been offered. Those "treatment-resistant" T2DM patients are a high-risk group for renal failure, therefore, a better understanding of the group and development of effective treatments are keen issues. However, to the best of our knowledge, no previous report focused on the group was found. Accordingly, we assessed "treatment-resistant" T2DM patients based on the hypothesis that either psychiatric disorders or psychosocial problems might underlie poor adherence, and then performed psychiatric interventions. As of December 2012, 28 patients who had HbA1C (NGSP) $\geq 9.0 \%$ for at least earlier 3 blood sampling or had been referred as resistant patients by their physician were psychiatrically assessed and treated by a psychiatrist qualified as an internist. The results of assessment of the total 28 patients showed $13(46 \%)$ cases of borderline intelligence, $12(43 \%)$ cases of binge-eating disorder, $8(29 \%)$ cases of bipolar disorder and $3(10 \%)$ cases of social anxiety disorder. These rates were three to eight times higher than prevalence of those disorders in the general population. Fifteen of the 28 patients were provided with medical/psychiatric intervention, and $\mathrm{HbA1C}$ levels decreased significantly within six to twelve months in 7 of them. To achieve long-term, good glycemic control in those patients, greater efficacy may be achieved by applying psychiatric pharmacotherapy and psychotherapy in parallel with medical therapy.

\section{WITHDRAWN}

\section{CLINICAL THERAPEUTICS/NEW TECHNOLOGY- GLUCOSE MONITORING AND SENSING}

WITHDRAWN

2519-PO

\section{Markers of $\boldsymbol{\beta}$-Cell Degradation}

2520-P0 KIM V. ANDREASSEN, SARA T. HJULER, LIPING LIU, OINLONG ZHENG, MORTEN A. KARSDAL, KIM HENRIKSEN, Herlev, Denmark, Beijing, China

Within recent years it has become increasingly clear that the loss of $\beta$-cells is an important feature of type 2 diabetes. Importantly, loss of $\beta$-cells appears to be a key pathological factor in the onset of T2D, and the protection against loss of $\beta$-cells is attractive from a treatment point-of-view. Hence monitoring this process is of substantial interest; however, presently available techniques do not provide the opportunity to specifically monitoring loss or degradation rates of $\beta$-cells.

We aimed at developing biomarkers of $\beta$-cell loss using the protein fingerprint technique, a technique focused on developing specific ELISA assays detecting enzyme-generated pathological fragments of proteins related to a specific pathology. To generate $\beta$-cell specific fragments we combined either the extracellular MMPs or the intracellular caspases with $\beta$-cell selective proteins, such as neuroligin-2, neurexin $1 \alpha$, GAD2, GLUT2 and insulin, and identified a series of fragments specific for either protein.

Monoclonal antibodies against these antibodies were raised and used to generate ELISAs specifically recognizing these cleavage products. Using extracts of rat pancreas we showed that the antibodies specifically recognized pancreatic tissue, indicating pathological relevance of the cleavage products.

In conclusion, $\beta$-cell loss is a highly relevant feature for both the early identification and the optimal treatment of T2D. By using the protein fingerprint technology we present data indicating that enzyme-generated fragments of $\beta$-cell-specific proteins may be useful as biomarkers of $\beta$-cell loss. Such markers may in the future assist in selecting the most optimal treatment for T2D patients, that now is recognized to be a heterogeneous group of patients that are warranted both optimal and differentiated personalized health care.

Supported by: Ministry of Science, Technology and Education (Denmark) 